\title{
A systematic comparison of the structural and dynamic properties of commonly used water models for molecular dynamics simulations
}

Sachini P. Kadaoluwa Pathirannahalage, ${ }^{\mathrm{a}, \mathrm{b}, \dagger}$ Nastaran Meftahi,,${ }^{c, \dagger}$ Aaron Elbourne, ${ }^{\mathrm{a}}$ Alessia C. G. Weiss, ${ }^{\mathrm{d}}$ Chris F. McConville, ${ }^{\mathrm{a}, \mathrm{e}}$ Agilio Padua, ${ }^{\mathrm{b}}$ David A. Winkler, ${ }^{\mathrm{f}}$ Margarida Costa Gomes, ${ }^{\mathrm{b}}$ Tamar L. Greaves, ${ }^{a}$ Tu C. Le, ${ }^{\mathrm{g}, *}$ Quinn A. Besford, ${ }^{\mathrm{d}, *}$ and Andrew J. Christofferson ${ }^{\mathrm{a}, *}$

${ }^{a}$ School of Science, RMIT University, Melbourne, Victoria 3000, Australia

${ }^{\mathrm{b}}$ Laboratoire de Chimie, Ecole Normale Supérieure de Lyon and CNRS, Lyon, France

c ARC Centre of Excellence in Exciton Science, School of Science, RMIT University, Melbourne, Victoria 3000, Australia

${ }^{d}$ Leibniz-Institut für Polymerforschung e.V., Hohe Straße 6, 01069 Dresden, Germany

e Institute for Frontier Materials, Deakin University, Geelong, Victoria 3220, Australia

${ }^{\mathrm{f}}$ School of Biochemistry and Genetics, La Trobe Institute for Molecular Science, La Trobe University, Bundoora, VIC 3086, Australia

Monash Institute of Pharmaceutical Sciences, Monash University, Parkville, VIC 3052, Australia School of Pharmacy, University of Nottingham, Nottingham NG7 2QL, UK

CSIRO Data61, Pullenvale, QLD 4069, Australia

g School of Engineering, RMIT University, Melbourne, Victoria 3001, Australia

* Corresponding authors: tu.le@rmit.edu.au, besford@ipfdd.de, andrew.christofferson@rmit.edu.au

$\dagger$ These authors contributed equally

\section{Keywords}

Molecular dynamics simulations, water models, machine learning, force field comparison, solvent structure

\begin{abstract}
Water is a unique solvent that is ubiquitous in biology and present in a variety of solutions, mixtures, and materials settings. It therefore forms the basis for all molecular dynamics simulations of biological phenomena, as well as for many chemical, industrial, and materials investigations. Over the years, many water models have been developed, and it remains a challenge to find a single water model that accurately reproduces all experimental properties of water simultaneously. Here, we report a comprehensive comparison of structural and dynamic properties of 30 commonly used 3-point, 4-point, 5-point, and polarizable water models simulated using consistent settings and analysis methods. For the properties of density, coordination number, surface tension, dielectric constant, self-diffusion coefficient, and solvation free energy of methane, models published within the past two decades consistently show better agreement with experimental values compared to models published earlier, albeit with some notable exceptions. However, no single model reproduced all experimental values exactly, highlighting the need to carefully choose a water model for a particular study, depending on the phenomena of interest. Finally, machine learning algorithms quantified the relationship between the water model force field parameters and the resulting bulk properties, providing insight into the parameter-property relationship and illustrating the challenges of developing a water model that can accurately reproduce all properties of water simultaneously.
\end{abstract}




\section{Introduction}

Water is commonly referred to as the universal solvent because it dissolves more substances than any other known liquid. It is also essential for life and understanding and simulating its role in all biological processes is extremely important. The exceptional solvating capacity of water is thought to result from its unique molecular properties, including high dielectric constant, hydrogen bonding structures, mobility, self-ionization, and strong permanent dipole moment. ${ }^{1}$ The combination of these unique properties allows water to facilitate many solvation and self-assembly phenomena. As such, it is a critical component of molecular dynamics (MD) simulations of proteins, ${ }^{2-5} \mathrm{DNA}^{6},{ }^{6-8}$ membranes, ${ }^{9-12}$ polymers, ${ }^{13-16}$ in solvent mixtures involving water, ${ }^{17-19}$ and water-solvated materials. ${ }^{20-25} \mathrm{MD}$ simulations are a powerful tool for providing atomistic insight into experimental systems of interest. Moreover, these techniques are accessible to researchers with little prior experience in modeling or computational approaches ${ }^{26}$ due to the development of freely available force field parameterization tools such as CGenFF, ${ }^{27,}{ }^{28}$ LigParGen ${ }^{29}$ and others, ${ }^{30,31}$ as well as tools for constructing systems and input files such as VMD ${ }^{32}$ and CHARMM-GUI. ${ }^{33}$ While this is undeniably of great benefit to the scientific community at large, the number and diversity of water models currently available (Figure 1) means it is critical that new users are aware of the potential pitfalls and concerns regarding the choice of water model for their MD simulations. For example, reproducing the correct dielectric constant of the individual components is essential for correctly describing the properties of a solvent mixture, ${ }^{17}$ and reproducing the surface tension of water is necessary for describing surfactants, monolayers, and interfaces. $^{34,35}$ For transport properties such as hydrogen bond lifetimes and viscosity, a reasonable value of the self-diffusion coefficient of water is desirable. ${ }^{36}$ To ensure that simulated systems involving water are reflective of real-world phenomena, it is crucial to validate that the chosen model can reproduce the intrinsic properties of water.

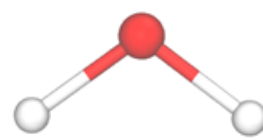

3-point

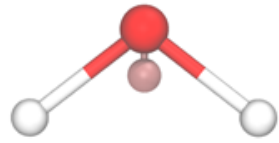

4-point

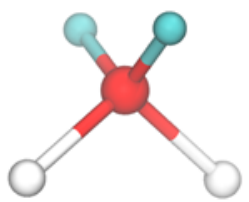

5-point

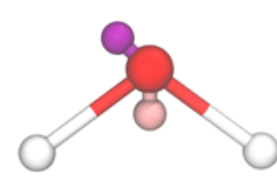

Polarizable (Drude)

Figure 1. Examples of water model geometry for the water models examined in this work. Oxygen and hydrogen are colored red and white, respectively. The offset partial charge on oxygen, $M$, in the 4-point models is colored pink. The lone pairs in the 5-point models, L, are colored cyan. The Drude oscillator in the polarizable model is colored purple.

Here, we use the term 'water model' to describe the complete set of force field parameters (i.e. bond lengths, angles, force constants for flexible models, atomic partial charges, and Lennard-Jones sigma and epsilon values that describe van der Waals interactions) necessary to perform MD simulations of water. While simulations of water have been reported since the $1960 \mathrm{~s},{ }^{37,38}$ the first generation of water models that are still commonly in use today was developed in the 1980s. The Simple Point Charge (SPC) water model, with a geometry based on the tetrahedral structure of water in ice and published by Berendsen et al. in $1981,{ }^{39}$ is typically used with the GROningen MOlecular Simulation (GROMOS) force field. ${ }^{40}$ The Transferable Interaction Potential 3-point model (TIP3P), with a geometry based on gas-phase water and published by Jorgensen et al. in 1983, ${ }^{41}$ is still used with both the Assisted Model Building with Energy Refinement (AMBER) ${ }^{42}$ and Optimized Potentials for Liquid Simulations $(\mathrm{OPLS})^{43}$ force fields. The slight differences in bond lengths and angles allow for SPC-based models to reproduce the experimentally observed second peak in the $\mathrm{O}-\mathrm{O}$ radial distribution function, whereas the second peak is missing entirely from the initial TIP3P model. Radial distribution functions, which reflect molecular structure in terms of density, are commonly used for comparison as they can be directly compared to experimental data extracted from X-ray scattering of molecular fluids. ${ }^{44}$ Further modifications to the TIP3P water model, including the addition of Lennard-Jones potentials to the hydrogens, resulted in the CHARMM TIP3P water model ${ }^{45}$ (also referred to as mTIP3P or TIPS3P, and 
referred to as TIPS3P hereafter in this work) which is used with the Chemistry at HARvard Macromolecular Mechanics (CHARMM) force field. ${ }^{46}$ In the same 1983 paper as the original TIP3P model, Jorgensen et al. also described a 4-point model, TIP4P, where the negative charge of the oxygen was offset from the Lennard-Jones potential, resulting in improved structural properties. ${ }^{41}$ The TIP4P water model is also commonly used with the OPLS force field. For the Consistent-Valence Force Field (CVFF), the CVFF water model has a geometry similar to TIP3P and non-bond parameters similar to SPC. It was developed concurrently with the rest of the force field, and is notable for having a flexible geometry, the use of a Morse potential for the $\mathrm{O}-\mathrm{H}$ bond, and cross-terms to reproduce vibrational frequencies. ${ }^{47}$

These models remain a popular choice due to their simple geometry, low computational cost, and relative accuracy. With the exception of CVFF, these models have a rigid geometry, which enables the use of a larger (e.g. 2 fs) timestep in MD simulations. While these models reproduced some experimental properties of water reasonably well, limited computational resources available at the time necessitated small system sizes of 125 to 216 water molecules, short simulation times of less than 20 ps, and short cut-offs of non-bond interactions typically between 7.5 and $8.5 \AA$. Moreover, many structural properties, such as densities and dielectric constants, were calculated using Monte Carlo, rather than MD, simulations. Monte Carlo simulations rely on statistical mechanics rather than dynamics, and may not reproduce the collective motion of molecules the same way as MD simulations. ${ }^{48}$

In the 1990s, Class II force fields based on ab initio potential energy surfaces were developed. ${ }^{49}$ Like $\mathrm{CVFF}$, they include cross terms to better reproduce experimental vibrational frequencies, and in contrast to Class I force fields, use a 9-6 rather than 12-6 functional form for the Lennard-Jones interactions (Equations S1 and S2, Supporting Information). The Condensed-phase Optimized Molecular Potentials for Atomistic Simulation Studies (COMPASS) ${ }^{50}$ and Polymer Consistent Force Field (PCFF) ${ }^{51}$ force fields are the two Class II force fields most commonly still in use, primarily for polymers and materials applications. ${ }^{52-56}$ Like CVFF, these force fields were developed to be used with commercial software, but the force field parameters for the COMPASS and PCFF water models were published in 2012 as part of the INTERFACE force field. ${ }^{20}$

While many of these water models performed well at $25^{\circ} \mathrm{C}$, few could accurately reproduce the temperature of maximum density of water of $4{ }^{\circ} \mathrm{C} .{ }^{57}$ In 2000 , Mahoney et al. published the 5-point TIP5P water model, which reproduced the density of water in the temperature range of -37 to $100{ }^{\circ} \mathrm{C}$ and a pressure range of 1 to $10,000 \mathrm{~atm} .{ }^{58}$ Following the initial publication of these 3-point, 4-point, and 5-point models, many modifications have been made to improve the properties based on new computational techniques and/or improvements in computing power. One of the first modifications to the initial model resulted in the SPC/E water model,,$^{59}$ which improved the reproduction of intermolecular potential energy. In order to increase compatibility with Ewald summation techniques for electrostatic interactions,${ }^{60}$ the TIP3P-Ew, ${ }^{61}$ TIP4P-Ew,${ }^{62}$ and TIP5P-Ew ${ }^{63}$ models were developed in 2004. In 2005, TIP4P was reparametrized to reproduce experimental properties with MD settings more in line with those currently used today, resulting in the TIP4P/2005 model. ${ }^{64}$ Flexible versions of the rigid models were also created, including SPC/Fw, ${ }^{65} \mathrm{TIP} 3 \mathrm{P} / \mathrm{Fw}^{66}$ and TIP4P/2005f.${ }^{67}$ In 2006 , the polarizable Simple Water Model with 4 sites and Negative Drude Polarizability (SWM4-NDP) was published. ${ }^{68}$ Other models were produced with the goal of maximizing a single property. The TIP4P/Ice model ${ }^{69}$ was developed to study the properties of ice, while the TIP $4 \mathrm{P} / \varepsilon,{ }^{70} \mathrm{SPC} / \varepsilon,{ }^{71} \mathrm{H} 2 \mathrm{O}-\mathrm{DC},{ }^{72}$ and $\mathrm{FBA} / \varepsilon^{73}$ models were all developed to reproduce the experimental dielectric constant. The SPC/Eb model $^{74}$ was developed to improve translational and rotational diffusion. The TIP4P-D mode ${ }^{75}$ was parameterized to better reproduce London dispersion interactions, and subsequently modified for the a99SBdisp force field for protein simulations. ${ }^{4}$

In the last decade, further additional generalized water models have been produced. The TIP3P-FB and TIP4P-FB models, ${ }^{76}$ based on the ForceBalance approach, were published in 2014, and updated as the TIP3P-ST and TIP4P-ST models ${ }^{77}$ to include surface tension in the optimization procedure in 2019. In 2018 an update of TIP5P was published as TIP5P-2018. ${ }^{78}$ In 2014, the "optimal" point charge 4-point 
model (OPC) was published,${ }^{79}$ followed by a 3-point variant (OPC3) in $2016,{ }^{80}$ where it was suggested that the accuracy limit of a 3-point rigid non-polarizable model had been reached.

Given the sheer number of available water models and reported properties under a variety of disparate conditions (e.g., number of molecules, simulation time, non-bond cutoffs, simulation method, etc), choosing an appropriate water model for a particular study can be a daunting task, particularly for those new to MD simulation techniques. It is therefore desirable to have a comparison of water models where the properties were determined under the same conditions for every model. Here, we report the density, coordination number, surface tension, static dielectric constant, self-diffusion coefficient, and free energy of hydrophobic solvation of 30 representative 3-point, 4-point, and 5-point water models, and one polarizable model (Table 1) using the open-source MD software LAMMPS and settings commonly used for simulations of biomolecules and materials today. We chose the SWM4-NDP model as a representative polarizable model because tools such as CHARMM-GUI and polarizer.py ${ }^{81}$ allow for the creation of LAMMPS input files for this model quickly and easily, even for non-expert users. This work serves as an update to the 2002 water model comparison by Guillot, ${ }^{82}$ and encompasses models published both before and after. While we don't examine continuum solvent models (i.e. implicit solvent $)^{83,84}$ or coarse-grained models, we point the reader to a comprehensive review of water models published in 2017 by Onufriev and Izadi for further reading. ${ }^{85}$

Table 1. Water models examined in this work.

\begin{tabular}{|c|c|c|c|c|c|}
\hline Name & Type & Flexible? & $\begin{array}{c}\text { Lennard-Jones } \\
\text { on H? }\end{array}$ & Year Published & Reference \\
\hline SPC & 3-point & No & No & 1981 & 39 \\
\hline TIP3P & 3-point & No & No & 1983 & 41 \\
\hline TIP4P & 4-point & No & No & 1983 & 41 \\
\hline TIPS3P & 3-point & No & Yes & 1985 & 45 \\
\hline $\mathrm{SPC} / \mathrm{E}$ & 3-point & No & No & 1987 & 59 \\
\hline CVFF & 3-point & Yes & No & 1988 & 47 \\
\hline PCFF & Class II & Yes & Yes & $1994^{\mathrm{a}}$ & 51 \\
\hline COMPASS & Class II & Yes & Yes & $1998^{b}$ & 50 \\
\hline TIP3P/Fw & 3-point & Yes & No & 1999 & 66 \\
\hline TIP5P & 5-point & No & No & 2000 & 58 \\
\hline TIP3P-Ew & 3-point & No & No & 2004 & 61 \\
\hline TIP4P-Ew & 4-point & No & No & 2004 & 62 \\
\hline TIP5P-Ew & 5-point & No & No & 2004 & 63 \\
\hline TIP4P/2005 & 4-point & No & No & 2005 & 64 \\
\hline TIP4P/Ice & 4-point & No & No & 2005 & 69 \\
\hline $\mathrm{SPC} / \mathrm{Fw}$ & 3-point & Yes & No & 2006 & 65 \\
\hline SWM4-NDP & Polarizable & No & No & 2006 & 68 \\
\hline TIP4P/2005f & 4-point & Yes & No & 2011 & 67 \\
\hline $\mathrm{TIP} 4 \mathrm{P} / \varepsilon$ & 4-point & No & No & 2014 & 70 \\
\hline OPC & 4-point & No & No & 2014 & 79 \\
\hline TIP3P-FB & 3-point & No & No & 2014 & 76 \\
\hline TIP4P-FB & 4-point & No & No & 2014 & 76 \\
\hline TIP4P-D & 4-point & No & No & 2015 & 75 \\
\hline $\mathrm{SPC} / \varepsilon$ & 3-point & No & No & 2015 & 71 \\
\hline OPC3 & 3-point & No & No & 2016 & 80 \\
\hline a99SB-disp & 4-point & No & No & 2018 & 4 \\
\hline TIP5P-2018 & 5-point & No & No & 2018 & 78 \\
\hline TIP3P-ST & 3-point & No & No & 2019 & 77 \\
\hline TIP4P-ST & 4-point & No & No & 2019 & 77 \\
\hline $\mathrm{FBA} / \varepsilon$ & 3-point & Yes & No & 2020 & 73 \\
\hline
\end{tabular}

${ }^{a}$ PCFF water force field parameters were published as part of the INTERFACE force field. ${ }^{20}$

${ }^{\mathrm{b}}$ Approximate COMPASS water parameters without cross-terms were published in 2012 as part of the INTERFACE force field. ${ }^{20}$ 
Finally, we used machine learning methods to explore the contributions of the parameters (model type, partial charge, Lennard-Jones parameters, bond length and angle) to the surface tension, dielectric constant, and self-diffusion coefficient. Machine learning can be used to predict a wide variety of material, ${ }^{86-89}$ chemical,,${ }^{90-93}$ and biological properties,,${ }^{94-97}$ with several commercial and non-commercial open-source platforms that can be used to develop machine learning algorithms such as Schrödinger, ${ }^{98}$ SYBYL,${ }^{99}$ TensorFlow (Google), ${ }^{100}$ and BioPPSy. ${ }^{101}$

\section{Methods}

Molecular dynamics simulations. All simulations were performed with the MD code Large-scale Atomic/Molecular Massively Parallel Simulator (LAMMPS) release 5Jun19. ${ }^{102}$ LAMMPS was chosen as it can run Class I, Class II, and polarizable models easily, with input files generated by CHARMMGUI, fftool, ${ }^{103}$ or Visual Molecular Dynamics (VMD). ${ }^{32}$ Moreover, LAMMPS provides the option of running 4-point water models as explicit 3-point models with the offset partial charge of the oxygen calculated internally. This allows 4-point models to be used from structure files that contain only 3point water (referred to hereafter as implicit 4-point simulations). LAMMPS is compatible with the PyLAT.py analysis tool, which facilitates the direct calculation of self-diffusion coefficients, dielectric constants, and radial distribution functions, among other properties. ${ }^{104}$

Initial configurations of 2,000 water molecules in a cubic box with approximate dimensions of $40 \times 40$ $\times 40 \AA^{3}$ were constructed using PACKMOL version $18.169,{ }^{105}$ and converted to LAMMPS data files using TopoTools ${ }^{106}$ release 1.7 in VMD version 1.9.3. All 3-point and implicit 4-point models used the same initial configuration, with a conjugate gradient energy minimization step in LAMMPS to optimize the initial geometry for each model, using force constants of $500 \mathrm{kcal} / \AA^{2}$ for the O-H bond and 50 $\mathrm{kcal} / \mathrm{radian}^{2}$ for the $\mathrm{H}-\mathrm{O}-\mathrm{H}$ angle with the rigid models, while flexible models used their default parameters. Explicit 4-point models, which were necessary for the calculation of dielectric constants, were constructed individually using PACKMOL, as were the 5-point models. The polarizable SWM4NDP model was constructed as an explicit 4-point model using PACKMOL, with the Drude oscillator added by the Python tool polarizer.py. ${ }^{81}$ The full set of force field parameters for the water models employed in this study are shown in Table 2 and Table 3 for 3-point and 4-point models, respectively, Table 4 for 5 -point and the polarizable model, and Table 5 for the force field parameters for flexible models.

Table 2. Force field parameters for 3-point models.

\begin{tabular}{|c|c|c|c|c|c|c|}
\hline Model & $\begin{array}{c}\text { O sigma } \\
(\AA)\end{array}$ & $\begin{array}{c}\text { O epsilon } \\
\text { (kcal/mol) }\end{array}$ & $\begin{array}{l}\text { O charge } \\
\text { (e) }\end{array}$ & $\begin{array}{c}\text { H charge } \\
\text { (e) }\end{array}$ & $\begin{array}{c}\text { O-H bond } \\
(\AA)\end{array}$ & $\begin{array}{c}\mathrm{H}-\mathrm{O}-\mathrm{H} \text { angle } \\
\left({ }^{\circ}\right)\end{array}$ \\
\hline SPC & 3.166 & 0.15535 & -0.82 & 0.41 & 1.0 & 109.466667 \\
\hline TIP3P & 3.15061 & 0.1521 & -0.834 & 0.417 & 0.9572 & 104.52 \\
\hline \multirow[t]{2}{*}{ TIPS3P } & 3.1506 & 0.1521 & -0.834 & 0.417 & 0.9572 & 104.52 \\
\hline & H: 0.4 & H: 0.046 & & & & \\
\hline SPC/E & 3.166 & 0.15535 & -0.8476 & 0.4238 & 1.0 & 109.466667 \\
\hline CVFF & 3.16552 & 0.155416 & -0.82 & 0.41 & 0.96 & 104.5 \\
\hline \multirow[t]{2}{*}{ PCFF } & 3.608 & 0.274 & -0.7982 & 0.3991 & 0.97 & 103.7 \\
\hline & H: 1.098 & H: 0.013 & & & & \\
\hline \multirow[t]{2}{*}{ COMPASS } & 3.84 & 0.08 & -0.82 & 0.41 & 0.9572 & 104.52 \\
\hline & H: 1.087 & H: 0.008 & & & & \\
\hline TIP3P/Fw & 3.1506 & 0.1522 & -0.834 & 0.417 & 0.96 & 104.5 \\
\hline TIP3P-Ew & 3.188 & 0.102 & -0.83 & 0.415 & 0.9572 & 104.52 \\
\hline $\mathrm{SPC} / \mathrm{Fw}$ & 3.165492 & 0.155425 & -0.82 & 0.41 & 1.012 & 113.24 \\
\hline TIP3P-FB & 3.178 & 0.155865 & -0.84844 & 0.42422 & 1.0118 & 108.15 \\
\hline $\mathrm{SPC} / \varepsilon$ & 3.1785 & 0.168704 & -0.89 & 0.445 & 1.0 & 109.45 \\
\hline OPC3 & 3.17427 & 0.163406 & -0.89517 & 0.447585 & 0.97888 & 109.47 \\
\hline TIP3P-ST & 3.19257 & 0.143858 & -0.85112 & 0.42556 & 1.023 & 108.11 \\
\hline $\mathrm{FBA} / \varepsilon$ & 3.1776 & 0.18937 & -0.845 & 0.4225 & 1.027 & 114.7 \\
\hline
\end{tabular}


Table 3. Force field parameters for 4-point models.

\begin{tabular}{|c|c|c|c|c|c|c|c|}
\hline Model & $\begin{array}{c}\text { O sigma } \\
(\mathbf{\AA})\end{array}$ & $\begin{array}{c}\text { O epsilon } \\
(\mathbf{k c a l} / \mathbf{m o l})\end{array}$ & $\begin{array}{c}\mathbf{M} \text { charge } \\
(\mathbf{e})^{\mathbf{a}}\end{array}$ & $\begin{array}{c}\text { H charge } \\
(\mathbf{e})\end{array}$ & $\begin{array}{c}\text { O-H bond } \\
(\mathbf{\AA})\end{array}$ & $\begin{array}{c}\text { H-O-H angle } \\
(\boldsymbol{\circ})\end{array}$ & $\begin{array}{c}\text { O-M bond } \\
(\mathbf{\AA})\end{array}$ \\
\hline TIP4P & 3.15365 & 0.155 & -1.04 & 0.52 & 0.9572 & 104.52 & 0.15 \\
\hline TIP4P-Ew & 3.16435 & 0.16275 & -1.04844 & 0.52422 & 0.9572 & 104.52 & 0.125 \\
\hline TIP4P/2005 & 3.1589 & 0.185207 & -1.1128 & 0.5564 & 0.9572 & 104.52 & 0.1546 \\
\hline TIP4P/Ice & 3.1668 & 0.210839 & -1.1794 & 0.5897 & 0.9572 & 104.52 & 0.1577 \\
\hline TIP4P/2005f & 3.1644 & 0.185207 & -1.1128 & 0.5564 & 0.9419 & 107.4 & 0.15555 \\
\hline TIP4P/ $\varepsilon$ & 3.165 & 0.18481 & -1.054 & 0.527 & 0.9572 & 104.52 & 0.105 \\
\hline OPC & 3.16655 & 0.212801 & -1.3582 & 0.6791 & 0.8724 & 103.6 & 0.1594 \\
\hline TIP4P-FB & 3.1655 & 0.179082 & -1.05174 & 0.52587 & 0.9572 & 104.52 & 0.10527 \\
\hline TIP4P-D & 3.165 & 0.223841 & -1.16 & 0.58 & 0.9572 & 104.52 & 0.1546 \\
\hline a99SB-disp & 3.165 & 0.238764 & -1.18 & 0.59 & 0.9572 & 104.52 & 0.1546 \\
\hline TIP4P-ST & 3.1661 & 0.176936 & -1.04344 & 0.52172 & 0.9572 & 104.52 & 0.0989 \\
\hline
\end{tabular}

${ }^{a}$ In 4-point models the oxygen center of mass carries a charge of 0 , and the charge is offset at point $\mathrm{M}$ (Figure 1).

Table 4. Force field parameters for 5-point and polarizable models.

\begin{tabular}{|c|c|c|c|c|c|c|c|c|c|}
\hline $\begin{array}{l}\text { 5-point } \\
\text { model }\end{array}$ & $\begin{array}{c}\text { O sigma } \\
(\AA)\end{array}$ & $\begin{array}{c}\text { O epsilon } \\
\text { (kcal/mol) }\end{array}$ & $\begin{array}{l}\text { O charge } \\
\text { (e) }\end{array}$ & $\begin{array}{l}\text { H charge } \\
\text { (e) }\end{array}$ & $\begin{array}{l}\text { L charge } \\
\text { (e) }\end{array}$ & $\begin{array}{c}\text { O-H bond } \\
(\AA)\end{array}$ & $\begin{array}{c}\mathrm{H}-\mathrm{O}-\mathrm{H} \\
\text { angle } \\
\left({ }^{\circ}\right)\end{array}$ & $\begin{array}{c}\text { O-L } \\
\text { bond } \\
(\AA)\end{array}$ & $\begin{array}{c}\mathrm{L}-\mathrm{O}-\mathrm{L} \\
\text { angle }\left({ }^{\circ}\right)\end{array}$ \\
\hline TIP5P & 3.12 & 0.16 & 0.0 & 0.241 & -0.241 & 0.9572 & 104.52 & 0.70 & 109.47 \\
\hline TIP5P-Ew & 3.097 & 0.178 & 0.0 & 0.241 & -0.241 & 0.9572 & 104.52 & 0.70 & 109.47 \\
\hline TIP5P-2018 & 3.145 & 0.188815 & -0.641114 & 0.394137 & -0.07358 & 0.9572 & 104.52 & 0.70 & 109.47 \\
\hline $\begin{array}{c}\text { Polarizable } \\
\text { model }\end{array}$ & $\begin{array}{c}\text { O sigma } \\
(\AA)\end{array}$ & $\begin{array}{c}\text { O epsilon } \\
\text { (kcal/mol) }\end{array}$ & $\begin{array}{l}\text { O charge } \\
\text { (e) }\end{array}$ & $\begin{array}{c}\text { H charge } \\
\text { (e) }\end{array}$ & $\begin{array}{l}\text { M charge } \\
\text { (e) }\end{array}$ & $\begin{array}{c}\text { Drude } \\
\text { charge (e) }\end{array}$ & $\begin{array}{c}\mathrm{O}-\mathrm{H} \\
\text { bond } \\
(\AA)\end{array}$ & $\begin{array}{c}\mathrm{H}-\mathrm{O}-\mathrm{H} \\
\text { angle } \\
\text { ( }\end{array}$ & $\begin{array}{c}\text { O-M } \\
\text { bond }(\AA)\end{array}$ \\
\hline SWM4-NDP & 3.18395 & 0.210939 & 1.71636 & 0.55733 & -1.11466 & -1.71636 & 0.9572 & 104.52 & 0.24034 \\
\hline
\end{tabular}

Table 5. Additional force field parameters for flexible models.

\begin{tabular}{|c|c|c|c|c|c|c|c|c|}
\hline Model & $\begin{array}{l}\mathbf{K}_{\mathrm{b}} \\
\left(\mathrm{kcal} \mathrm{mol}^{-1} \AA^{-2}\right)\end{array}$ & $\begin{array}{l}\mathrm{K}_{\theta} \\
\left(\mathrm{kcal} \mathrm{mol}^{-1} \mathrm{rad}^{-2}\right)\end{array}$ & & & & & & \\
\hline CVFF & 540.6336 & 50.0 & & & & & & \\
\hline TIP3P/Fw & 529.581 & 34.0435 & & & & & & \\
\hline $\mathrm{SPC} / \mathrm{FW}$ & 529.581 & 37.95 & & & & & & \\
\hline $\mathrm{FBA} / \varepsilon$ & 358.509 & 45.77 & & & & & & \\
\hline Model & $K_{2 b}$ & $K_{3 b}$ & $K_{4 b}$ & $K_{2 \theta}$ & $K_{3 \theta}$ & $K_{4 \theta}$ & $\mathbf{K}_{\mathbf{b b}}$ & $K_{b \theta}$ \\
\hline PCFF & 563.28 & -1428.22 & 1902.12 & 49.84 & -11.6 & -8.0 & -9.5 & 22.35 \\
\hline COMPASS & 552.0 & -1276.0 & 1787.0 & 46.65 & -11.7 & -8.79 & & \\
\hline Model & D (kcal/mol) & $\alpha$ & $\mathbf{K}_{\boldsymbol{\theta}}$ & & & & $\mathbf{K}_{\mathbf{b b}}$ & $\mathbf{K}_{\mathbf{b} \boldsymbol{\theta}}$ \\
\hline $\operatorname{CVFF}(\mathrm{cm})^{\mathrm{a}}$ & 104.0 & 2.28 & 50.0 & & & & -14.5 & 31.3 \\
\hline TIP4P/2005f & 103.389340 & 2.287 & 43.95435 & & & & & \\
\hline
\end{tabular}

a The CVFF has two variations: a standard flexible model, and a model with cross terms and a Morse potential for the $\mathrm{O}-\mathrm{H}$ bond. The latter is referred to here as the $\mathrm{CVFF}(\mathrm{cm})$ model.

For the 3-point and implicit 4-point simulations, initial random velocities with a Gaussian distribution at $1 \mathrm{~K}$ were assigned to each atom, and $500 \mathrm{ps}$ of MD simulation was performed in the NVT (constant volume, constant temperature) ensemble with the system heated linearly from $1 \mathrm{~K}$ to $298.15 \mathrm{~K}$. This was followed by 500 ps of NVT simulation at $298.15 \mathrm{~K}$, and $4 \mathrm{~ns}$ of NPT (constant pressure, constant temperature) simulation at $298.15 \mathrm{~K}$ and $1.0 \mathrm{~atm}$. Production runs were performed for $20 \mathrm{~ns}$, with simulation snapshots saved every $10 \mathrm{ps}$.

Densities and radial distribution functions (RDFs) were determined from the 20 ns NPT simulations. Following this, the simulation box length was set to the average value from the NPT simulations and additional NVT simulations were run in triplicate for $20 \mathrm{~ns}$. These simulations were used for the calculation of coordination number, self-diffusion coefficient, and dielectric constant using PyLAT.py. For 5-point and polarizable models, PyLAT.py did not calculate the correct self-diffusion coefficient, and for these models the calculation was performed using the mean-squared displacement of oxygen 
atoms from LAMMPS and the Einstein relation. ${ }^{107}$ For the calculation of coordination number, it was necessary to output trajectories containing only atoms (i.e. no lone pairs) for the 5-point models. For the calculation of dielectric constant, it was necessary to run additional NVT simulations with explicit 4-point models, as the implicit 4-point model trajectories would not produce the correct dipole moments for 4-point models. PyLAT.py also failed to calculate the correct dielectric constant for the polarizable model, which we calculated with our in-house code. For the determination of surface tension, the box was extended to $120 \AA$ in the $z$ dimension and NVT simulations were run for each model, with the surface tension determined from the pressure tensors calculated in LAMMPS by the virial method. ${ }^{108}$

The solvation free energy of model solutes into each water model can be probed using Widom's test particle insertion method. ${ }^{109,110}$ The solute is inserted into an equilibrated configuration of the pure solvent at position $\boldsymbol{x}_{\mathrm{s}}$. The change in the potential energy $\Delta U$ upon insertion, without allowing the system to relax, is then calculated. For MD simulations in the $N p T$ ensemble, the solvation free energy, $\Delta G_{s o l}$, equivalent to the excess chemical potential, is given by

$$
\Delta G_{\text {sol }}=-k_{B} T \log \left(\frac{1}{V}\left\langle\int_{V} d x_{\mathrm{s}} \exp \left(-\frac{\Delta U}{k_{B} T}\right)\right\rangle\right)
$$

Where $\langle\ldots\rangle$ represents the ensemble average, $V$ the volume of the simulation cell. The simplest numerical scheme for approximating the integral in Equation 1 is by integrating over an evenly spaced grid of insertion points. ${ }^{111} \mathrm{~A}$ regular grid for $\boldsymbol{x}_{\mathrm{s}}$ with a mesh spacing of about 1 bohr was chosen. A successful insertion was defined as an insertion that gives a non-zero $\exp \left(-\frac{\Delta U}{k_{B} T}\right)$, evaluated from the Lennard-Jones potential. The solute-water cross parameters were deduced from the Lorentz-Berthelot mixing rules, where $\varepsilon_{i j}=\sqrt{\varepsilon_{i i} \varepsilon_{j j}}$, and $\sigma_{i j}=\left(\sigma_{i i}+\sigma_{j j}\right) / 2$. At least 1000 particle insertions were performed for every other snapshot of each NPT simulation trajectory (i.e., 20 ps between snapshots, 1,000 snapshots total). As the Class II models COMPASS and PCFF use a 9-6 functional form for the Lennard-Jones interactions, 12-6 parameters were extrapolated for the solute-solvent interactions from energy vs. distance plots of the 9-6 functional. For the PCFF model, the values were $3.155 \AA$ for sigma and $0.274 \mathrm{kcal} / \mathrm{mol}$ for epsilon for the oxygen, and $0.96 \AA$ for sigma and $0.013 \mathrm{kcal} / \mathrm{mol}$ for epsilon for the hydrogen. For the COMPASS model, the values were $3.355 \AA$ for sigma and $0.0 .08 \mathrm{kcal} / \mathrm{mol}$ for epsilon for the oxygen, and $0.95 \AA$ for sigma and $0.008 \mathrm{kcal} / \mathrm{mol}$ for epsilon for the hydrogen.

Machine learning models. We used QSPR (quantitative structure property relationship) ${ }^{112}$ algorithms to explore the quantitative relationship between the force field parameters of the water models in this study and the dielectric constant, diffusion coefficient and surface tension. In a conventional QSPR machine learning technique, descriptors are mathematical values which can define the physical and chemical properties of compounds in dataset. In this study, we considered six force field parameters of the water models as descriptors: $\mathrm{O}$ sigma, $\mathrm{O}$ epsilon, $\mathrm{O}$ charge, $\mathrm{O}-\mathrm{H}$ bond length, $\mathrm{H}-\mathrm{O}-\mathrm{H}$ angle, $\mathrm{M}-\mathrm{O}$ distance. Also, to consider the effect of 3-point, 4-point, and flexible models on the resultant properties, we applied a 1-hot binary matrix, where the descriptor was given a value of 1 if present and 0 if absent. A schematic of these descriptors is provided in Figure S1, Supporting Information. To generate the quantitative relationship between dielectric constant, diffusion coefficient and surface tension with water models, we employed the linear algorithm MLREM (Multiple Linear Regression with Expectation Maximization) $)^{113}$ implemented in the CSIRO-BioModeller package. ${ }^{114-116}$ To evaluate the quality of models the correlation coefficient between experimental and calculated values (by machine learning) for the data set $\left(\mathrm{R}^{2}\right)$ were computed. The closer the $\mathrm{R}^{2}$ value was to 1.0, the better the model. In addition to $\mathrm{R}^{2}$ values, the standard error of estimation (SEE) was calculated, where SEE represents the root mean square error between the calculated and measured values of data points, adjusted for degrees of freedom. ${ }^{117}, 118$ Thus, the SEE is a more robust description of model quality than the $\mathrm{R}^{2}$ value. ${ }^{119}$ 


\section{Results and discussion}

Results for density, coordination number, surface tension, dielectric constant, and self-diffusion coefficient are presented in Table 6 , and the percent difference between the calculated and experimental value is shown in Figure S2, Supporting Information. For the settings employed in this work, the OPC model provided the best average agreement with experimental data for these properties, while the $\mathrm{TIP} 4 \mathrm{P} / \varepsilon$ and TIP4P-FB models were the only models with all properties within $10 \%$ of experimental values. Subdividing the data by model type, the OPC3, OPC, TIP5P, and SPC/Fw models provided the best agreement for 3-point, 4-point, 5-point, and flexible models, respectively. The only polarizable model examined in this work, SWM4-NDP, also performed reasonably well, with an average difference from experimental values of less than $10 \%$. Although many models were not specifically parameterized or tested for all of the properties examined here, we note a general improvement in agreement with experimental values as a function of publication year.

Table 6. Calculated properties of water models using consistent simulation settings. Uncertainties are reported as standard deviations except for surface tension, where the uncertainty is reported as standard error.

\begin{tabular}{|c|c|c|c|c|c|c|}
\hline Model & $\begin{array}{l}\text { Density } \\
\left(\mathrm{g} / \mathrm{cm}^{3}\right)\end{array}$ & $\begin{array}{c}\text { Coordination } \\
\text { number }\end{array}$ & $\begin{array}{l}\text { Surface } \\
\text { tension } \\
(\mathrm{mN} / \mathrm{m}) \\
\end{array}$ & $\begin{array}{c}\text { Dielectric } \\
\text { constant }\end{array}$ & $\begin{array}{c}\text { Self-diffusion } \\
\text { coefficient } \\
\left(\times 10^{-5} \mathrm{~cm}^{2} / \mathrm{s}\right) \\
\end{array}$ & $\begin{array}{c}\Delta \mathbf{G}_{\text {methane }} \\
(\mathbf{k J} / \mathbf{m o l})\end{array}$ \\
\hline SPC & $0.972 \pm 0.006$ & $5.585 \pm 0.001$ & $50.3 \pm 0.2$ & $65 \pm 1$ & $4.32 \pm 0.04$ & $8.88 \pm 0.08$ \\
\hline TIP3P & $0.980 \pm 0.006$ & $6.239 \pm 0.001$ & $47.0 \pm 0.2$ & $95 \pm 3$ & $5.72 \pm 0.04$ & $8.51 \pm 0.08$ \\
\hline TIP4P & $0.994 \pm 0.006$ & $5.14 \pm 0.001$ & $52.2 \pm 0.2$ & $51.3 \pm 0.5$ & $2.57 \pm 0.04$ & $8.91 \pm 0.08$ \\
\hline TIPS3P & $1.007 \pm 0.006$ & $7.5 \pm 0.3$ & $51.1 \pm 0.2$ & $106 \pm 1$ & $5.55 \pm 0.06$ & $10.0 \pm 0.1$ \\
\hline $\mathrm{SPC} / \mathrm{E}$ & $0.993 \pm 0.006$ & $4.9 \pm 0.2$ & $57.6 \pm 0.2$ & $73 \pm 1$ & $2.60 \pm 0.03$ & $9.64 \pm 0.08$ \\
\hline CVFF & $0.978 \pm 0.006$ & $6.4 \pm 0.3$ & $47.3 \pm 0.4$ & $135 \pm 3$ & $5.95 \pm 0.05$ & $7.9 \pm 0.2$ \\
\hline CVFF $(\mathrm{cm})$ & $0.989 \pm 0.006$ & $5.761 \pm 0.001$ & $49.8 \pm 0.4$ & $151 \pm 2$ & $5.2 \pm 0.2$ & $8.9 \pm 0.1$ \\
\hline PCFF & $1.008 \pm 0.005$ & $13.5 \pm 0.4$ & $69.5 \pm 0.4$ & $159 \pm 2$ & $7.7 \pm 0.2$ & $11.9 \pm 0.2$ \\
\hline COMPASS & $0.958 \pm 0.006$ & $4.953 \pm 0.001$ & $40.7 \pm 0.4$ & $118 \pm 2$ & $6.4 \pm 0.1$ & $11.59 \pm 0.06$ \\
\hline TIP3P/Fw & $1.027 \pm 0.006$ & $5.3 \pm 0.3$ & $55.2 \pm 0.4$ & $197 \pm 2$ & $3.8 \pm 0.1$ & $9.38 \pm 0.09$ \\
\hline TIP5P & $0.985 \pm 0.006$ & $4.991 \pm 0.001$ & $48.9 \pm 0.2$ & $94 \pm 3$ & $2.79 \pm 0.03$ & $8.00 \pm 0.06$ \\
\hline TIP3P-Ew & $0.996 \pm 0.006$ & $4.7300 \pm 0.0004$ & $47.1 \pm 0.2$ & $92 \pm 1$ & $4.11 \pm 0.05$ & $9.38 \pm 0.09$ \\
\hline TIP4P-Ew & $0.996 \pm 0.005$ & $4.6900 \pm 0.0005$ & $59.2 \pm 0.2$ & $65 \pm 1$ & $2.54 \pm 0.01$ & $8.8 \pm 0.2$ \\
\hline TIP5P-Ew & $1.003 \pm 0.006$ & $5.143 \pm 0.001$ & $52.2 \pm 0.2$ & $100 \pm 2$ & $2.92 \pm 0.04$ & $8.25 \pm 0.03$ \\
\hline TIP4P/2005 & $0.997 \pm 0.005$ & $4.7053 \pm 0.0001$ & $63.5 \pm 0.2$ & $58 \pm 1$ & $2.18 \pm 0.04$ & $8.4 \pm 0.1$ \\
\hline TIP4P/Ice & $0.993 \pm 0.006$ & $4.634 \pm 0.001$ & $73.4 \pm 0.2$ & $63 \pm 2$ & $1.21 \pm 0.03$ & $7.93 \pm 0.07$ \\
\hline $\mathrm{SPC} / \mathrm{Fw}$ & $1.007 \pm 0.006$ & $4.743 \pm 0.001$ & $58.6 \pm 0.4$ & $80 \pm 3$ & $2.57 \pm 0.06$ & $8.99 \pm 0.05$ \\
\hline SWM4-NDP & $0.990 \pm 0.005$ & $5.209 \pm 0.001$ & $63.1 \pm 0.5$ & $75 \pm 1$ & $2.57 \pm 0.05$ & $9.4 \pm 0.2$ \\
\hline TIP4P/2005f & $0.996 \pm 0.005$ & $5.0 \pm 0.2$ & $60.3 \pm 0.4$ & $59 \pm 2$ & $2.76 \pm 0.04$ & $8.68 \pm 0.05$ \\
\hline TIP4P/\& & $0.996 \pm 0.006$ & $4.717 \pm 0.001$ & $64.6 \pm 0.2$ & $79 \pm 2$ & $2.16 \pm 0.01$ & $9.2 \pm 0.1$ \\
\hline OPC & $0.997 \pm 0.005$ & $5.1971 \pm 0.0005$ & $70.1 \pm 0.2$ & $78 \pm 1$ & $2.27 \pm 0.02$ & $9.34 \pm 0.09$ \\
\hline TIP3P-FB & $0.990 \pm 0.006$ & $4.6786 \pm 0.0004$ & $60.3 \pm 0.2$ & $79 \pm 4$ & $2.14 \pm 0.05$ & $8.39 \pm 0.06$ \\
\hline TIP4P-FB & $0.997 \pm 0.006$ & $4.702 \pm 0.001$ & $64.7 \pm 0.2$ & $77 \pm 1$ & $2.10 \pm 0.03$ & $9.9 \pm 0.1$ \\
\hline TIP4P-D & $0.993 \pm 0.006$ & $5.149 \pm 0.001$ & $70.8 \pm 0.2$ & $63 \pm 1$ & $2.01 \pm 0.07$ & $8.86 \pm 0.06$ \\
\hline$\overline{\mathrm{SPC} / \varepsilon}$ & $0.991 \pm 0.005$ & $4.672 \pm 0.001$ & $65.3 \pm 0.2$ & $80 \pm 3$ & $1.55 \pm 0.05$ & $9.12 \pm 0.09$ \\
\hline OPC3 & $0.991 \pm 0.006$ & $4.9 \pm 0.2$ & $61.0 \pm 0.2$ & $79 \pm 1$ & $2.28 \pm 0.02$ & $8.54 \pm 0.03$ \\
\hline a99SB-disp & $0.996 \pm 0.006$ & $5.173 \pm 0.001$ & $74.4 \pm 0.2$ & $67 \pm 1$ & $1.78 \pm 0.03$ & $9.20 \pm 0.03$ \\
\hline TIP5P-2018 & $0.997 \pm 0.006$ & $5.175 \pm 0.001$ & $61.6 \pm 0.2$ & $129 \pm 2$ & $2.31 \pm 0.09$ & $8.3 \pm 0.1$ \\
\hline TIP3P-ST & $0.993 \pm 0.005$ & $4.6009 \pm 0.0005$ & $63.8 \pm 0.2$ & $81 \pm 2$ & $1.24 \pm 0.02$ & $9.38 \pm 0.05$ \\
\hline TIP4P-ST & $0.999 \pm 0.006$ & $4.7034 \pm 0.0003$ & $64.5 \pm 0.2$ & $82 \pm 4$ & $2.02 \pm 0.01$ & $9.5 \pm 0.3$ \\
\hline FBA $/ \varepsilon$ & $0.991 \pm 0.005$ & $4.64 \pm 0.001$ & $68.0 \pm 0.4$ & $75 \pm 1$ & $1.56 \pm 0.02$ & $8.8 \pm 0.1$ \\
\hline Experimental & $0.997^{120}$ & $4.7^{121}$ & $71.99^{122}$ & $78.3^{123}$ & $2.30^{124}$ & $\mathbf{8 . 0 8 3}^{125}$ \\
\hline
\end{tabular}

One notable exception is the SPC/E model, which despite being published in 1987 still performs quite well under the conditions employed in this work. We emphasize here that we are not claiming to have found the "best" models, but rather for the system size and simulation settings we chose for this study, 
these are the models that present the best agreement with the experimental values we chose to compare to. We will discuss each property in more detail in the following sections.

Density. All models reproduced the experimental density of water reasonably well, with no model published after 2006 presenting a difference from experimental value above $0.7 \%$. This is perhaps unsurprising, as density is one of the properties almost every water model is parametrized to reproduce. Models published before 2000 tended to reproduce the experimental density value more poorly in this study, at least in part due to the use of the particle-particle particle-mesh (PPPM) ${ }^{126}$ treatment of longrange electrostatics used here. While PPPM and the related particle-mesh Ewald (PME) ${ }^{127}$ are commonly used in simulations today, many of the older water models were developed with a relatively short non-bond cutoff and no treatment of long-range electrostatics. The TIP3P-Ew, TIP4P-Ew, and TIP5P-Ew models, published in 2004, represent reparameterizations of the original TIP3P, TIP4P, and TIP5P models, respectively, for use with Ewald summation for long-range electrostatics. All three Ewald models improved the reproduction of experimental density without changing the water model geometry, with the TIP3P-Ew and TIP4P-Ew models changing both charge and Lennard-Jones parameters, and the TIP5P-Ew changing only the Lennard-Jones parameters. The COMPASS water model exhibited the greatest deviation from the experimental density in this study, but like the TIPfamily models this is most likely due to the PPPM treatment of long-range electrostatics. Indeed, a 2004 paper found the density error to be $0.34 \%$ when a group-based cutoff scheme was used, and $3.25 \%$ when an Ewald summation was used. ${ }^{128}$ Overall, these results suggest caution when combining older water models with current long-range electrostatic treatments like PPPM and PME if accurate reproduction of water density is desirable.

Coordination number. Water models published after 2000 exhibited a fairly narrow range of values for the average number of water molecules in the first coordination shell of a given water molecule, with a minimum value of 4.6 for the TIP3P-ST water model, and a maximum of 5.2 for the SWM4NDP polarizable water model (Figure 2). We chose an experimental value of 4.7 for comparison, which was based on X-ray scattering data and supported by molecular dynamics simulations with a polarizable force field. ${ }^{121}$

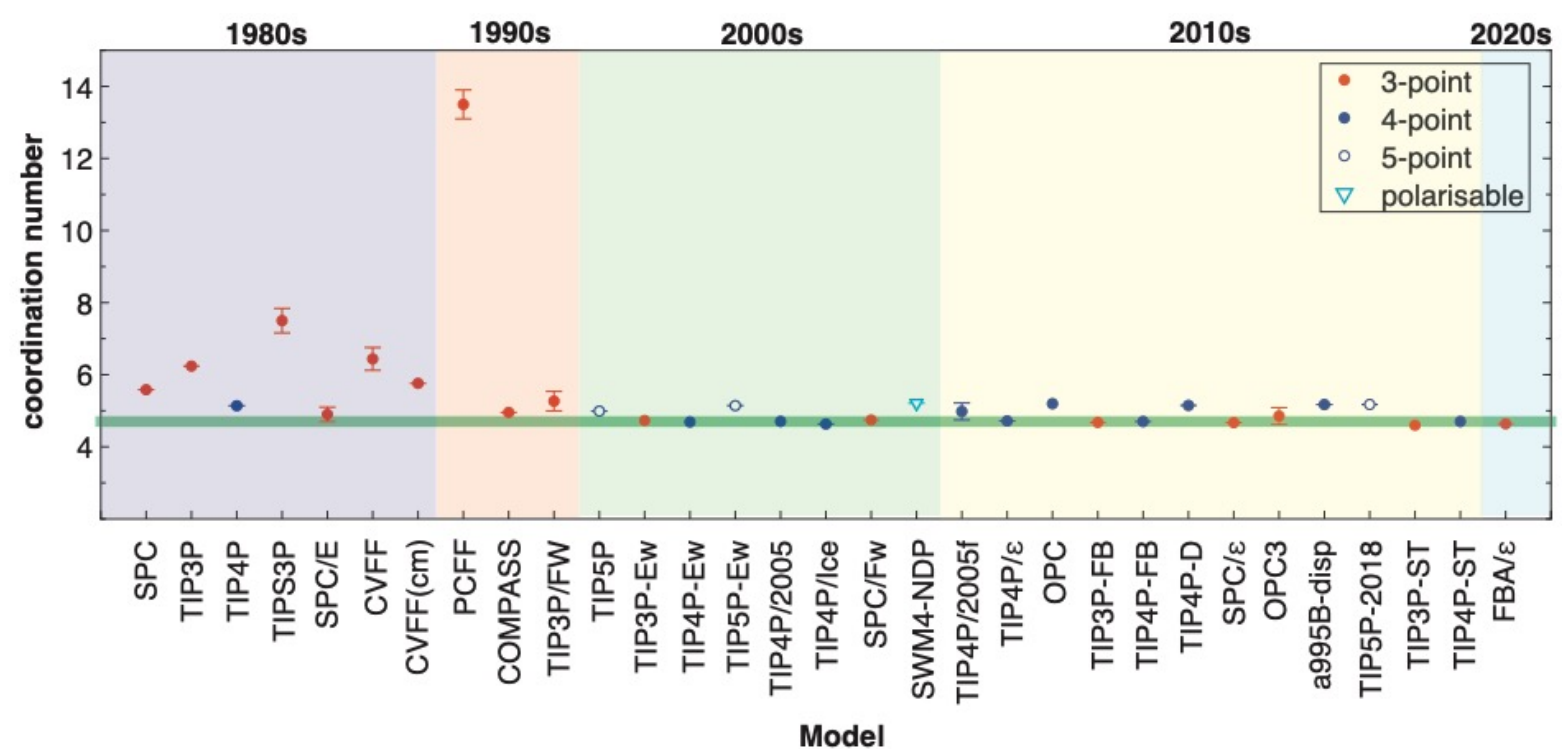

Figure 2. Coordination number of the first solvation shell for each water model. The horizontal green line corresponds to the experimental coordination number of 4.7. ${ }^{121}$

The PCFF water model exhibited the greatest deviation from the experimental value, with an average of $13.5 \pm 0.4$ water molecules in the first coordination shell. Indeed, the O-O RDF for PCFF is significantly different from the other water models (Figure S3, Supporting Information), with a much broader peak and first minimum at $4.6 \AA$, compared to $\sim 3.3-3.4 \AA$ for most other water models. As the coordination number is found by integrating to the first minimum in the RDF, this creates a significant 
difference in the calculated coordination number for the water molecule. However, despite this large difference, the O-O, O-H, and $\mathrm{H}-\mathrm{H}$ RDFs for PCFF are in general qualitatively similar to the other models. The PCFF force field, as part of the CFF force field family, ${ }^{129}$ was parameterized to reproduce the structure and vibrational frequencies of isolated molecules in the gas phase, ${ }^{128}$ not bulk properties, which may account for some of the observed discrepancies. The original SPC and TIP3P-based models (including CVFF) also produced larger coordination numbers compared to the experimental value. The SPC/E model provided greater agreement compared to SPC by changing the partial charges only, while TIP3P-Ew improved agreement by changing both partial charges and Lennard-Jones parameters. The $\mathrm{SPC} / \mathrm{Fw}$ provided greater agreement for flexible models by small changes to the Lennard-Jones parameters and changes to the water geometry, while retaining the original SPC partial charges.

Surface tension. Historically, surface tension was not considered as part of the target data in the force field parameterization process. It is therefore not surprising that water models published before 2005 exhibited poor agreement with the experimental surface tension (Figure 3). In contrast to density and coordination number, the Ewald correction to the TIP-family models did not significantly improve the surface tension. One notable exception was the PCFF force field, which had one of the closest values to experiment of any water model studied here. This is most likely due to the Lennard-Jones epsilon value for the oxygen, which is the largest of any water model in this study. It is known that epsilon values generally correlate positively with surface tension, and one force field parameterization strategy is to simply scale all epsilon values until the experimental surface tension is reproduced. ${ }^{130}$ However, this is not the only factor, as one of the best models for surface tension, TIP3P-ST, has one of the smallest epsilon values of any model tested in this work.

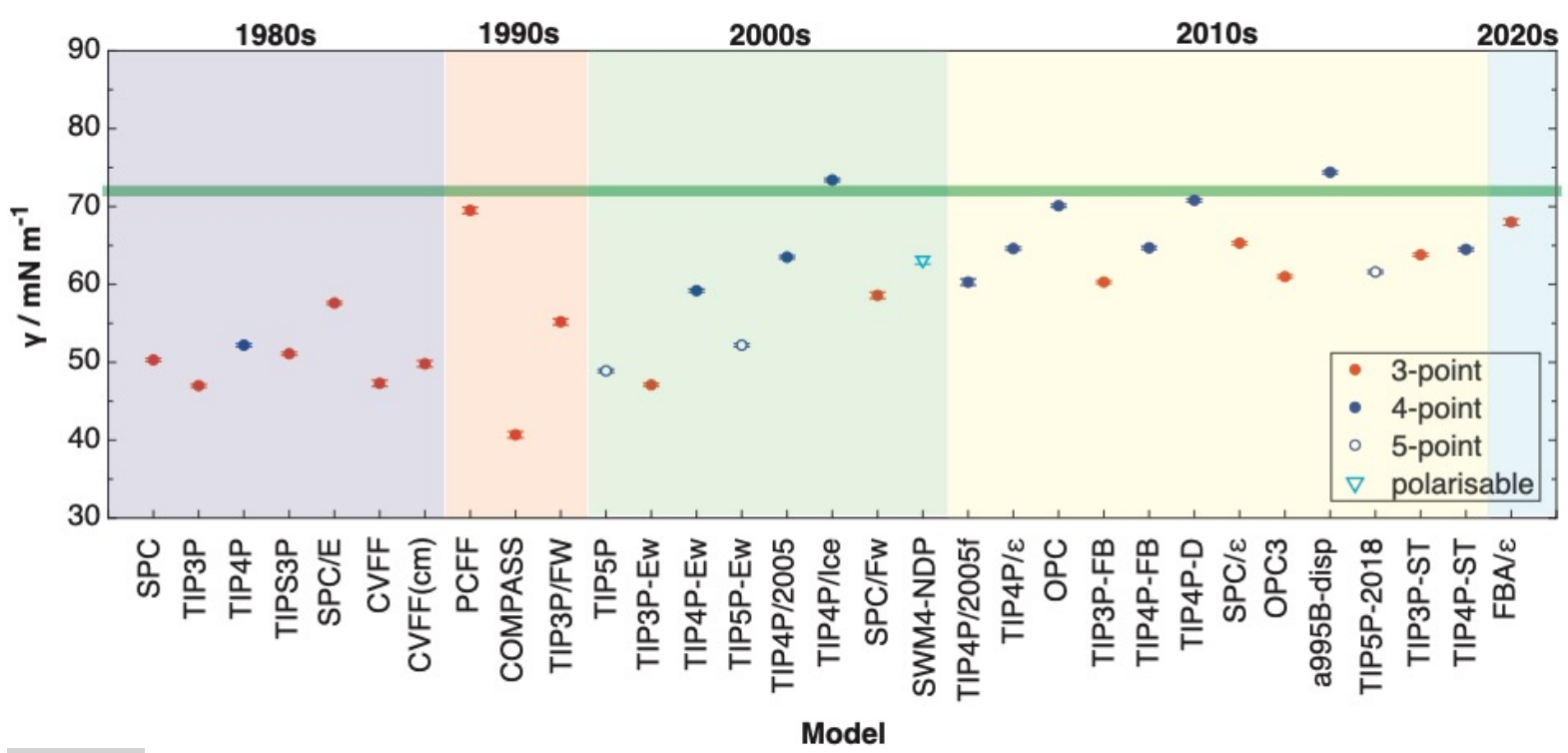

Figure 3. Surface tension at the liquid-vapor interface for each water model. The horizontal green line corresponds to the experimental surface tension of $71.99 \mathrm{mN} / \mathrm{m} .{ }^{122}$

The analytical tail correction to the surface tension due to the Lennard-Jones cutoff, which generally increases the value of the calculated surface tension by $\sim 3-4 \mathrm{mN} / \mathrm{m},{ }^{108}$ was not performed in this study, as it has been shown recently ${ }^{131}$ to perhaps overestimate the surface tension by $\sim 2 \mathrm{mN} / \mathrm{m}$. Nevertheless, the surface tension has been shown to increase as the Lennard-Jones cutoff is increased, and by extrapolating to an infinite cutoff, the TIP3P-ST model was found to have a surface tension within 1 $\mathrm{mN} / \mathrm{m}$ of the experimental value, ${ }^{77}$ while in this study it was found to be $\sim 8 \mathrm{mN} / \mathrm{m}$ below the experimental value. Therefore, despite appearing to systematically underestimate the surface tension (Figure 3), nearly all models published in 2005 or later can be considered to reproduce the experimental surface tension reasonably well, with the TIP4P/Ice and a99SB-disp overestimating the surface tension somewhat. Long-range corrections to the Lennard-Jones interactions, similar to long-range corrections to the electrostatics like PPPM and PME, may improve the calculated surface tension as well. The absence of ions and dissolved gas in the simulated systems could also contribute to a lower surface 
tension compared to experiment. ${ }^{132}$ For additional discussion of the use of surface tension as target data in force field parameterization, we refer the reader to the publication of the TIP3P-ST and TIP4P-ST models. ${ }^{77}$

Dielectric constant. Liquid water exhibits a high dielectric constant, $\varepsilon_{s}$, that is unique amongst most polar liquids. The macroscopic dielectric constant contains information on the collective orientational correlations between molecules and their permanent dipole moments, where the dielectric constant is directly linked to the strength of dipolar forces between water molecules. ${ }^{133}$ Computationally, the dielectric constant can be readily accessed from the total dipole moment of the system, $M$, via

$$
\varepsilon_{S}=1+\frac{\left\langle M^{2}\right\rangle-\langle M\rangle^{2}}{3 \varepsilon_{0} V k_{B} T}
$$

where $\varepsilon_{0}$ is the permittivity of free space, and $V$ the system volume. Since the dielectric constant results from the cooperative motion and structure between water molecules, it is one of the most important properties a water model should reproduce. ${ }^{134}$ The results for the dielectric constant are presented in Figure 4. As with the other properties discussed so far, there is a general improvement in the reproduction of the dielectric constant as a function of publication year. One notable exception is the TIP5P-2018 model, which exhibits a $63 \%$ overestimate of the experimental value, despite being one of the most recent models published. Designed to improve the density and self-diffusion coefficient over a range of temperatures, the TIP5P-2018 model does represent an improvement for 5-point models in self-diffusion coefficient, density, surface tension, and other properties not examined in this study, but the authors report that in order to reproduce the dynamic properties of water, it was necessary to downscale the charges, which resulted in an anomalously high dielectric constant. ${ }^{78}$

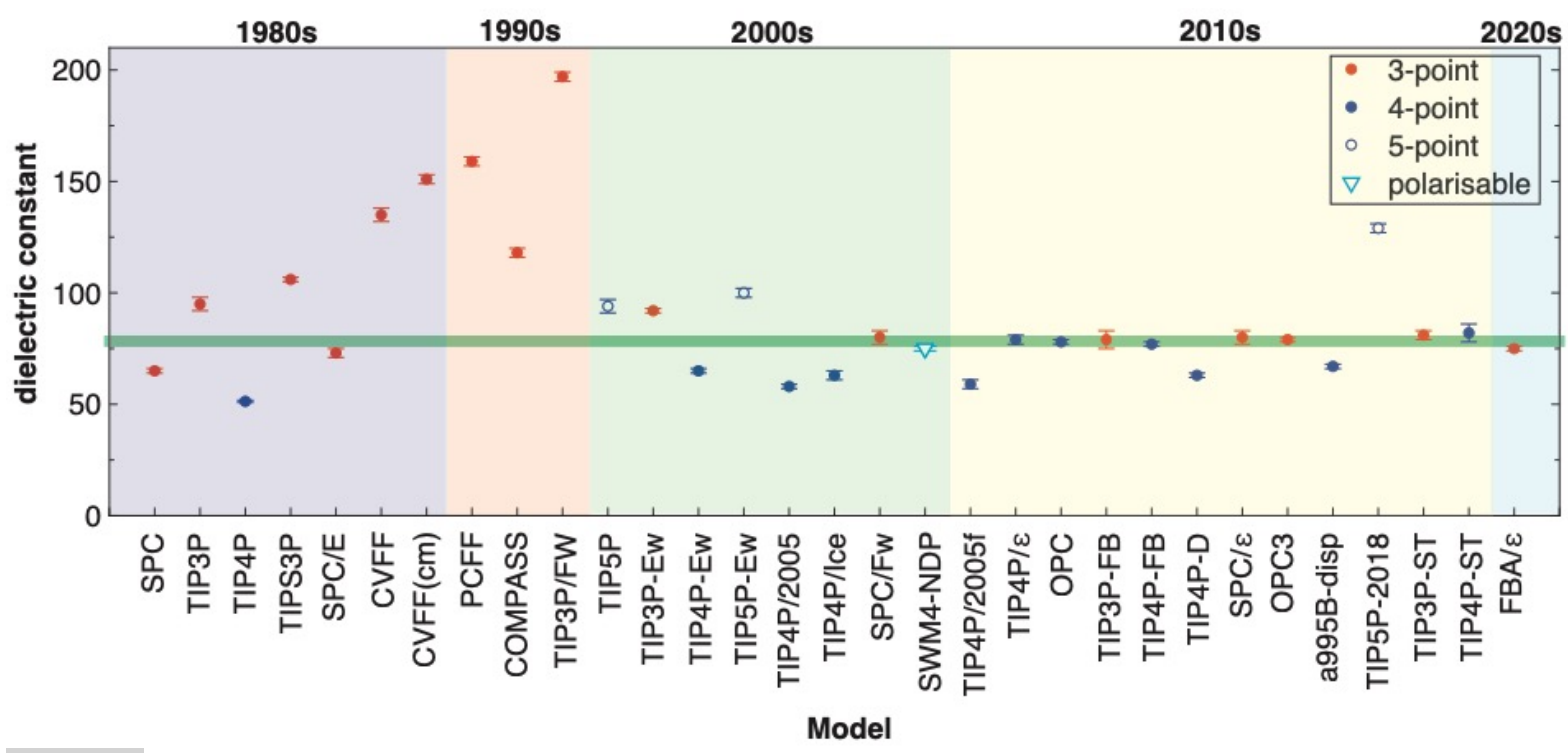

Figure 4. Static dielectric constant for each water model. The horizontal green line corresponds to the experimental dielectric constant of 78.3. ${ }^{123}$

In general, models published before 2006 reproduce the experimental dielectric constant poorly, with TIP3P, TIP5P, and Class II models overestimating and SPC and TIP4P models underestimating the dielectric constant. This is likely due to the fact that dielectric constant was generally not part of the target data in the force field parameterization process, rather than due to the use of PPPM for long-range electrostatics here. In fact, values for the dielectric constant for TIP4P and SPC/E calculated in the 1990s agree quite well with the values calculated in this work. ${ }^{135}$ On the other hand, values for TIP3P calculated in the 1990s varied from 73 to 82 using different methods, ${ }^{136,}{ }^{137}$ which while quite close to the experimental value differ significantly from the value calculated here. This further emphasizes the need for properties to be calculated within a consistent framework, using consistent methods. Three of the models in this study were specifically parameterized to reproduce the dielectric constant: $\mathrm{TIP} 4 \mathrm{P} / \varepsilon$, 
$\mathrm{SPC} / \varepsilon$, and the flexible $\mathrm{FBA} / \varepsilon$. As expected, all reproduce the experimental dielectric constant quite well, although for the $\mathrm{SPC} / \varepsilon$ and $\mathrm{FBA} / \varepsilon$ models it appears to come at the cost of the self-diffusion coefficient, which is discussed in the next section. For the SWM4-NDP polarizable model, the Drude induced dipole represents (in a very simplified manner) electronic degrees of freedom, and the polarization terms are expected to be relevant for the dielectric constant. It is therefore not surprising that it performs well for the reproduction of the experimental dielectric constant.

Self-diffusion coefficient. As with the other properties examined in this work, significant improvement in the reproduction of the experimental self-diffusion constant occurred from about 2005 onwards (Figure 5). Notable exceptions occurred where structuring was of higher priority, such as TIP4P/Ice (for better reproduction of the phase diagram), $\mathrm{SPC} / \varepsilon$ and $\mathrm{FBA} / \varepsilon$ (for better reproduction of the dielectric constant), and TIP3P-ST (for better reproduction of the surface tension). Conversely, the TIP5P-2018 model deliberately sacrificed good agreement with the experimental dielectric constant in order to better reproduce the experimental self-diffusion coefficient.

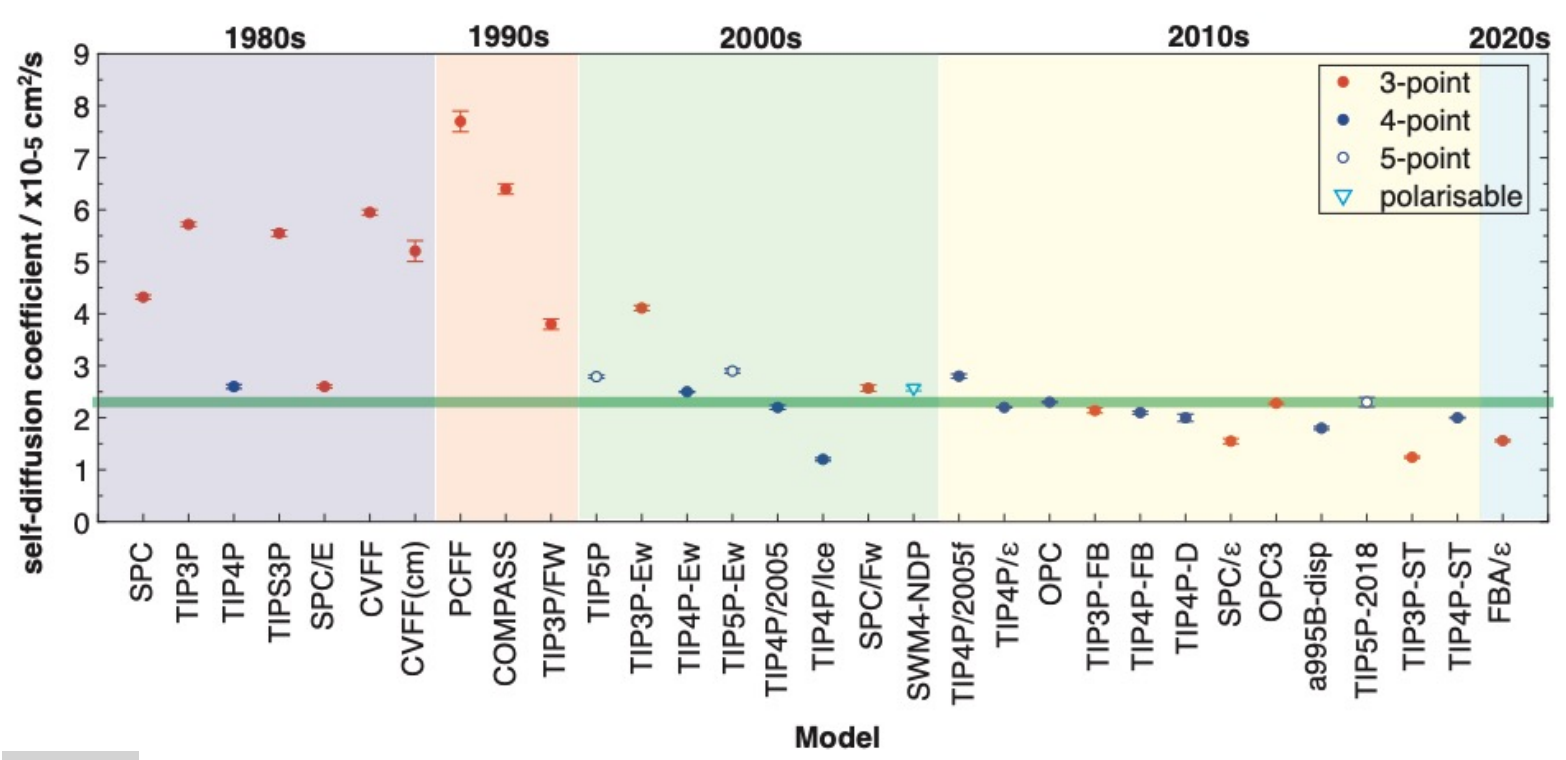

Figure 5. Self-diffusion coefficient for each water model. The horizontal green line corresponds to the experimental self-diffusion coefficient of $2.30 \times 10^{-5} \mathrm{~cm}^{2} / \mathrm{s} .{ }^{124}$

With the exception of TIP3P-FB, the TIP3P-based models consistently failed to reproduce the experimental self-diffusion coefficient of water. This is perhaps not surprising, as the original TIP3P model was developed for Monte Carlo, not MD, simulations, and the self-diffusion coefficient was not part of the parameterization process or reported in the original paper. ${ }^{41}$ The CVFF, PCFF, and COMPASS models also failed to reproduce the experimental self-diffusion coefficient. These models were parameterized to be used with the commercial simulation package Discover ${ }^{138}$ and indeed a selfdiffusion coefficient of $2.45 \times 10^{-5} \mathrm{~cm}^{2} / \mathrm{s}$ was reported for CVFF water when a quintic spline switching function (not currently available in LAMMPS) was used for the non-bonded interactions. ${ }^{139}$ While an anomalously high diffusion coefficient is not generally desirable, it can be used in some cases to deliberately speed up conformational sampling, ${ }^{15}, 16,25,140,141$ as an alternative to other advanced sampling methods. ${ }^{142-144}$ Self-diffusion coefficients depend on the size of the simulation box, and calculated values increase as the box size increases. ${ }^{145}$

Machine learning analysis of the parameter-property relationship. The main aim of using machine learning was to investigate and quantify the effect of the water model force field parameters on the resulting dielectric constant, diffusion coefficient, and surface tension, rather than prediction for models not studied here. Therefore, we did not partition the dataset into a training set and test set, and instead used the entire dataset as the training set. Because there were too few Class II, 5-point, or polarizable models in this study, only Class I 3-point and 4-point models were used for the machine learning analysis. This provided 24 water models for machine learning analysis, which was sufficient for surface 
tension, dielectric constant, and self-diffusion coefficient, but not for density, coordination number, or free energy of solvation (see below), as the range in values of these properties was too small.

Table 7. Performance of MLREM for surface tension, dielectric constant, and self-diffusion coefficient by applying $100 \%$ of the dataset to the training set. Scaled values are reported for standard error estimation.

\begin{tabular}{|l|c|c|}
\hline Property & $\mathbf{R}^{2}$ & Standard error estimation \\
\hline Surface tension & 0.95 & $0.084 \mathrm{mN} / \mathrm{m}$ \\
\hline Dielectric constant & 0.92 & 0.073 \\
\hline Self-diffusion coefficient & 0.92 & $0.108 \times 10^{-5} \mathrm{~cm}^{2} / \mathrm{s}$ \\
\hline
\end{tabular}

Table 7 presents the performance of MLREM for surface tension, dielectric constant, and self-diffusion coefficient. With only nine descriptors (oxygen sigma, epsilon, and charge; O-H bond; $\mathrm{H}-\mathrm{O}-\mathrm{H}$ angle; M-O distance; 3-point yes/no; 4-point yes/no; and flexible yes/no), the MLREM algorithm was able to describe the relationship between the water model parameters and resultant properties with an $\mathrm{R}^{2}$ greater than 0.9 and standard error estimation of $\sim 0.1$.
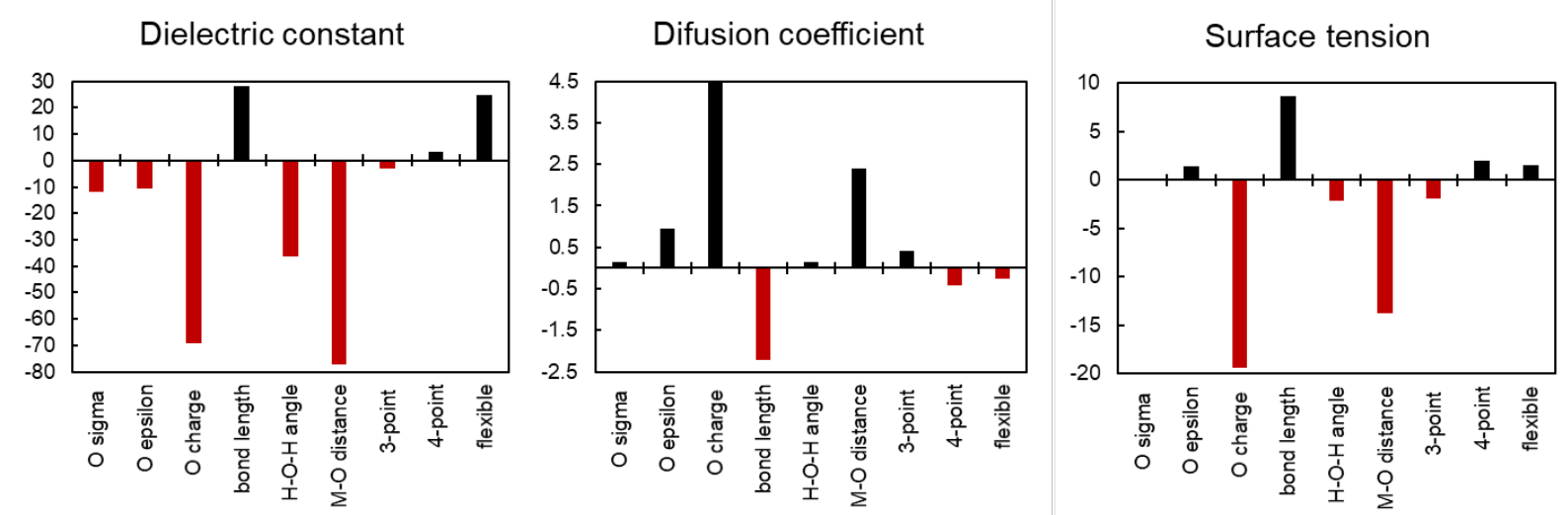

Figure 6. Scaled contributions of the water model force field parameters to the resulting properties.

Figure 6 presents the contribution as well as the effect of descriptors on each property, where the parameters with positive and negative sign led to an increase or decrease in the value of the property, respectively. By comparing these three graphs, it can be seen that the partial charge on the oxygen $(\mathrm{O}$ charge) has a significant effect on each property, with more negative values decreasing the surface tension and dielectric constant, and more positive values increasing the diffusion coefficient. Interestingly, the distance between the partial charge and the center of mass of the oxygen (M-O distance) had a large effect on all properties, with a shorter M-O distance associated with a lower surface tension and dielectric constant, and a longer $\mathrm{M}-\mathrm{O}$ distance associated with a greater self-diffusion coefficient. Critically, no single parameter simultaneously increased or decreased all properties, illustrating the difficulties in parameterizing a water model that reproduces all experimental properties well.

Particle insertion. Hydrophobic effects are considered to play an important role in solvation phenomena such as protein stability and other self-assembly phenomena. ${ }^{110}$ Therefore, it is important to validate the capacity of each water model to reproduce hydrophobic behavior. Here, we probe the solvation free energies of methane for each water molecule by particle insertion methods. ${ }^{109}$ 


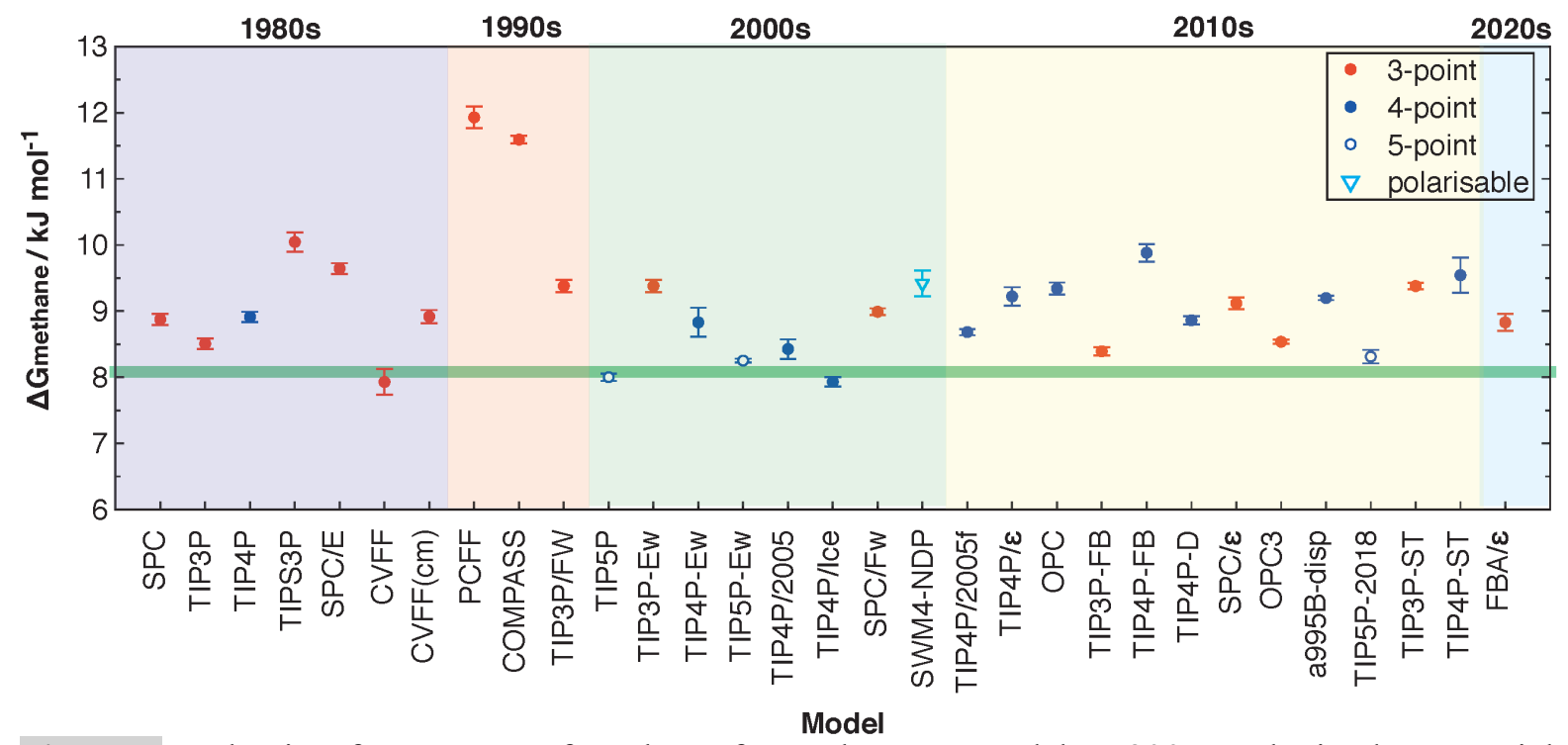

Figure 7. Solvation free energy of methane for each water model at $300 \mathrm{~K}$, obtained via particle insertion methods. The horizontal green line corresponds to the experimental solvation free energy of methane of $8.083 \mathrm{~kJ} / \mathrm{mol}^{125}$

The free energy of hydrophobic solvation for the insertion of the methane bead into each water model is presented in Figure 7. In general, most water models were within $2 \mathrm{~kJ} / \mathrm{mol}$ of the experimental value, although there was a systematic overestimation of the value. The Class II models were an exception, but this may be due to the fact that it was necessary to extrapolate 12-6 Lennard-Jones parameters for these models in order to perform particle insertion. Interestingly, all of the 5-point models as well as some models with poorer overall agreement with the structural and dynamic properties studied here such as TIP3P and CVFF reproduced the free energy of hydrophobic solvation reasonably well. TIP3P has been found previously to predict hydration free energies of a range of small neutral organic molecules with an rms error of $1.24 \mathrm{kcal} / \mathrm{mol} .{ }^{146}$ This further illustrates the difficulties in parameterizing a water model for all possible properties.

Timing. One final consideration in the choice of water model is the computational efficiency. The average timing for each water model type, reported in ns/day, when run on a single 48-CPU node (comprising two 24-core Intel Xeon Scalable 'Cascade Lake' processors) of the Australian National Computational Infrastructure supercomputer Gadi is listed in Table 8.

Table 8. Average timings for each water model type in ns/day.

\begin{tabular}{|l|c|}
\hline Model type & Average computational efficiency (ns/day) \\
\hline Rigid 3-point & 33.6 \\
\hline Flexible 3-point & 32.0 \\
\hline Class II & 29.7 \\
\hline Implicit 4-point & 18.1 \\
\hline Explicit 4-point & 18.6 \\
\hline 5-point & 16.4 \\
\hline Polarizable & 9.1 \\
\hline
\end{tabular}

In terms of computational efficiency, 3-point models were significantly faster to run than 4-point, 5point, or polarizable models. The additional force constants and cross-terms in the flexible and Class II models only slightly decreased their efficiency, as the most time-consuming step is the calculation of non-bonded interactions. For the 4-point models there was little difference, on average, between running them as explicit 4-point models or as 3-point models with an internal calculation for the offset partial charge on oxygen (implicit 4-point model). While all of the models in this study were run with a $1 \mathrm{fs}$ timestep, for the rigid non-polarizable models it is possible to run with a $2 \mathrm{fs}$ timestep, effectively 
doubling the computational efficiency reported here. The purpose of this study was to run all models under the same conditions, but presumably improvements in efficiency can be gained by fine-tuning the non-bond settings, utilizing GPUs, or other methods.

\section{Conclusions and outlook}

We present these results as a broad comparison of water models performed under consistent simulation conditions. We anticipate that this will be a useful reference for the properties of these models (some of which are not found elsewhere), and as a benchmarking and comparison dataset for future studies. We deliberately have not classified the models in terms of 'best' or 'worst', as the 'best' water model will depend on the specific application. This work also serves as an update to other excellent water model comparisons. ${ }^{82,147,148}$ For example, in 2011 Vega and Abascal performed a comprehensive comparison of water models for a variety of properties and ranked TIP4P/2005 as the best, ${ }^{149}$ but every 4-point model published since 2011 exhibits a better average agreement with experimental values for the structural and dynamic properties studied here.

While it is unfortunate that some of the water models exhibiting the poorest agreement with experimental properties are some of the ones most commonly used with biological and materials force fields today, it does not necessarily invalidate such studies. In the case of the CHARMM force field, traditionally all other molecules are parameterized relative to the CHARMM TIP3P (TIPS3P in this study) water model and subsequently adjusted to reproduce other experimental data, ${ }^{46}$ which should ameliorate some of the deficiencies in that model. The CHARMM developers themselves have tested adjustments to the water model to improve agreement with experimental data. ${ }^{150}$ Moreover, as discussed by Onufriev and Izadi ${ }^{85}$ water models that perfectly reproduce the bulk properties of water may in fact be poorer for some of the wide variety of hydrophobic and hydrophilic environments where water can be found, and the only solution is to carefully benchmark the system of interest to as much available experimental data as possible, regardless of the specific water model used. While the Class II force fields did not generally reproduce well the experimental properties examined in this study, these force fields are still potentially useful due to the variety of atom types available, transferrable nature of their parameters, and ability to reproduce other experimental phenomena. The PCFF force field, for example, was able to reproduce the triple helix stereocomplex of poly(methyl methacrylate) in agreement with experimental X-ray diffraction profiles. ${ }^{53,151}$ However, when used outside the commercial software they were parameterized for, Class II force fields should be used with caution, and may be better for comparison purposes between systems rather than for attempting to find absolute values. ${ }^{16,35,152}$

In the past decade, many studies have examined the feasibility of using some of the more recently developed water models with existing force fields. ${ }^{153,}{ }^{154}$ When done properly this can represent a significant advancement in the modeling of solvated systems, but it must be done with care. For example, it was found that when a force field that used arithmetic mixing rules for the Lennard-Jones interactions was mixed with ion parameters that were parameterized for geometric mixing rules, the salt spontaneously and unphysically crystalized from solution. ${ }^{155}$ Fortunately, new compatible ion parameters have been developed for the OPC, OPC3, TIP3P-FB, and TIP4P-FB water models. ${ }^{156-158}$ While the careful testing of newer water models with older force fields for biomaterials and other molecules is one solution, another current solution is to develop new force fields for compatibility with the more recently developed water models. ${ }^{4,159,160}$

Finally, while the accuracy limit may indeed have been reached for 3-point and 4-point non-polarizable models, other models for water are continually in development, such as the 7-point TIP7P, ${ }^{161}$ the reactive force field ReaxFF, ${ }^{162}$ the FFLUX polarizable and multipolar model, ${ }^{163}$ as well as others based on DFT and machine learning, ${ }^{164-166}$ to name a few. ${ }^{85,167}$ As computing power increases over the coming years, we expect that the ability to predict and reproduce the observed properties of water molecules will also continue to improve, which in turn will improve our understanding of this remarkable solvent. 


\section{Data and Software Availability}

The MD code LAMMPS is freely available at: https:/lammps.sandia.gov/. The analysis tool PyLAT.py is freely available at: https://github.com/MaginnGroup/PyLAT. The initial configuration generator PACKMOL is freely available at: http://leandro.iqm.unicamp.br/m3g/packmol/home.shtml. The visualization and modelling software VMD is freely available at: https://www.ks.uiuc.edu/Research/vmd/. The software for generating Drude polarizable models is freely available at: https://github.com/paduagroup/clandpol. Widom insertion can be performed using the fix widom command in LAMMPS. LAMMPS input files can also be freely generated directly by CHARMM-GUI: https://charmm-gui.org/. Multiple Linear Regression is available in many commercial and free software packages, including statsmodels (Python): https://www.statsmodels.org/stable/index.html. All files necessary to reproduce the simulations in this work may be found at: https://github.com/VisualizationAndModelProduction/WaterModelComparison

\section{Acknowledgements}

This research was undertaken with the assistance of supercomputing resources from the National Computational Infrastructure (NCI), which is supported by the Australian Government, under the National Computational Merit Allocation Scheme (project k159). This research was funded in part by the Alexander von Humboldt Foundation (Q.A.B.). The authors gratefully acknowledge Prof. Peter Daivis for helpful discussions.

\section{References}

1. Water and life: the unique properies of H2O. CRC Press: Boca Raton, FI, USA, 2010.

2. Karplus, M.; McCammon, J. A., Molecular dynamics simulations of biomolecules. Nature Structural Biology 2002, 9 (9), 646-652.

3. Christofferson, A. J., Asymmetric ligand binding in homodimeric Enterobacter cloacae nitroreductase yields the Michaelis complex for nitroaromatic substrates. J. Mol. Model. 2020, 26 (2), 28.

4. Robustelli, P.; Piana, S.; Shaw, D. E., Developing a molecular dynamics force field for both folded and disordered protein states. Proceedings of the National Academy of Sciences 2018, 115 (21), E4758.

5. Lindorff-Larsen, K.; Maragakis, P.; Piana, S.; Eastwood, M. P.; Dror, R. O.; Shaw, D. E., Systematic Validation of Protein Force Fields against Experimental Data. PLOS ONE 2012, 7 (2), e32131.

6. Beveridge, D. L.; Barreiro, G.; Suzie Byun, K.; Case, D. A.; Cheatham, T. E.; Dixit, S. B.; Giudice, E.; Lankas, F.; Lavery, R.; Maddocks, J. H.; Osman, R.; Seibert, E.; Sklenar, H.; Stoll, G.; Thayer, K. M.; Varnai, P.; Young, M. A., Molecular Dynamics Simulations of the 136 Unique Tetranucleotide Sequences of DNA Oligonucleotides. I. Research Design and Results on $\mathrm{d}(\mathrm{CpG})$ Steps. Biophysical Journal 2004, 87 (6), 3799-3813.

7. Christofferson, A.; Zhao, L.; Sun, H.; Huang, Z.; Huang, N., Theoretical studies of the base pair fidelity of selenium-modified DNA. Journal of Physical Chemistry B 2011, 115 (33), 10041-10048.

8. Mackerell, A. D.; Nilsson, L., Molecular dynamics simulations of nucleic acid-protein complexes. Current Opinion in Structural Biology 2008, 18 (2), 194-199.

9. Enkavi, G.; Javanainen, M.; Kulig, W.; Róg, T.; Vattulainen, I., Multiscale Simulations of Biological Membranes: The Challenge To Understand Biological Phenomena in a Living Substance. Chem. Rev. 2019, 119 (9), 5607-5774.

10. Leonard, A. N.; Wang, E.; Monje-Galvan, V.; Klauda, J. B., Developing and Testing of Lipid Force Fields with Applications to Modeling Cellular Membranes. Chem. Rev. 2019, 119 (9), 62276269.

11. Marrink, S. J.; Corradi, V.; Souza, P. C. T.; Ingólfsson, H. I.; Tieleman, D. P.; Sansom, M. S. P., Computational Modeling of Realistic Cell Membranes. Chem. Rev. 2019, 119 (9), 6184-6226. 
12. Venable, R. M.; Krämer, A.; Pastor, R. W., Molecular Dynamics Simulations of Membrane Permeability. Chem. Rev. 2019, 119 (9), 5954-5997.

13. Zhelavskyi, O. S.; Kyrychenko, A., Atomistic molecular dynamics simulations of the LCST conformational transition in poly(N-vinylcaprolactam) in water. J. Mol. Graph. Model. 2019, 90, 5158.

14. Kishinaka, S.; Morita, A.; Ishiyama, T., Molecular structure and vibrational spectra at water/poly(2-methoxyethylacrylate) and water/poly(methyl methacrylate) interfaces: A molecular dynamics simulation study. J. Chem. Phys. 2019, 150 (4), 044707.

15. Truong, V. K.; Bhadra, C. M.; Christofferson, A. J.; Yarovsky, I.; Al Kobaisi, M.; Garvey, C. J.; Ponamoreva, O. N.; Alferov, S. V.; Alferov, V. A.; Tharushi Perera, P. G.; Nguyen, D. H. K.; Buividas, R.; Juodkazis, S.; Crawford, R. J.; Ivanova, E. P., Three-Dimensional Organization of SelfEncapsulating Gluconobacter oxydans Bacterial Cells. ACS Omega 2017, 2 (11), 8099-8107. 16. Christofferson, A. J.; Elbourne, A.; Cheeseman, S.; Shi, Y.; Rolland, M.; Cozzolino, D.; Chapman, J.; McConville, C. F.; Crawford, R. J.; Wang, P.-Y.; Truong, N. P.; Anastasaki, A.; Truong, V. K., Conformationally tuned antibacterial oligomers target the peptidoglycan of Gram-positive bacteria. J. Colloid Interface Sci. 2020, 580, 850-862.

17. de Jesús-González, N. E.; Pérez de la Luz, A.; López-Lemus, J.; Alejandre, J., Effect of the Dielectric Constant on the Solubility of Acetone in Water. Journal of Chemical \& Engineering Data 2018, 63 (5), 1170-1179.

18. Yalcin, D.; Christofferson, A. J.; Drummond, C. J.; Greaves, T. L., Solvation properties of protic ionic liquid - molecular solvent mixtures. Phys. Chem. Chem. Phys. 2020.

19. Dalgicdir, C.; Rodríguez-Ropero, F.; van der Vegt, N. F. A., Computational Calorimetry of PNIPAM Cononsolvency in Water/Methanol Mixtures. J. Phys. Chem. B 2017, 121 (32), 7741-7748.

20. Heinz, H.; Lin, T.-J.; Kishore Mishra, R.; Emami, F. S., Thermodynamically Consistent Force Fields for the Assembly of Inorganic, Organic, and Biological Nanostructures: The INTERFACE Force Field. Langmuir 2012, 29 (6), 1754-1765.

21. Wright, L. B.; Rodger, P. M.; Corni, S.; Walsh, T. R., GolP-CHARMM: First-Principles Based Force Fields for the Interaction of Proteins with $\mathrm{Au}(111)$ and $\mathrm{Au}(100)$. J. Chem. Theory Comput. 2013, 9 (3), 1616-1630.

22. Zhang, W.; Besford, Q. A.; Christofferson, A. J.; Charchar, P.; Richardson, J. J.; Elbourne, A.; Kempe, K.; Hagemeyer, C. E.; Field, M. R.; McConville, C. F.; Yarovsky, I.; Caruso, F., CobaltDirected Assembly of Antibodies onto Metal-Phenolic Networks for Enhanced Particle Targeting. Nano Letters 2020, 20 (4), 2660-2666.

23. Besford, Q. A.; Christofferson, A. J.; Kalayan, J.; Sommer, J.-U.; Henchman, R. H., The Attraction of Water for Itself at Hydrophobic Quartz Interfaces. J. Phys. Chem. B 2020, 124 (29), 6369-6375.

24. Christofferson, A. J.; Al-Garawi, Z. S.; Todorova, N.; Turner, J.; Del Borgo, M. P.; Serpell, L. C.; Aguilar, M.-I.; Yarovsky, I., Identifying the Coiled-Coil Triple Helix Structure of $\beta$-Peptide Nanofibers at Atomic Resolution. ACS Nano 2018.

25. Zhang, W.; Christofferson, A. J.; Besford, Q. A.; Richardson, J. J.; Guo, J.; Ju, Y.; Kempe, K.; Yarovsky, I.; Caruso, F., Metal-dependent inhibition of amyloid fibril formation: synergistic effects of cobalt-tannic acid networks. Nanoscale 2019, 11 (4), 1921-1928.

26. Kinnaman, L. J.; Roller, R. M.; Miller, C. S., Comparing Classical Water Models Using Molecular Dynamics To Find Bulk Properties. J. Chem. Educ. 2018, 95 (5), 888-894.

27. Vanommeslaeghe, K.; MacKerell, A. D., Automation of the CHARMM General Force Field (CGenFF) I: Bond Perception and Atom Typing. J. Chem. Inf. Model 2012, 52 (12), 3144-3154.

28. Vanommeslaeghe, K.; Raman, E. P.; MacKerell, A. D., Automation of the CHARMM General Force Field (CGenFF) II: Assignment of Bonded Parameters and Partial Atomic Charges. J. Chem. Inf. Model 2012, 52 (12), 3155-3168. 
29. Dodda, L. S.; Cabeza de Vaca, I.; Tirado-Rives, J.; Jorgensen, W. L., LigParGen web server: an automatic OPLS-AA parameter generator for organic ligands. Nucleic Acids Research 2017, 45 (W1), W331-W336.

30. Mayne, C. G.; Saam, J.; Schulten, K.; Tajkhorshid, E.; Gumbart, J. C., Rapid parameterization of small molecules using the force field toolkit. J. Comput. Chem. 2013, 34 (32), 2757-2770.

31. Kumar, A.; Yoluk, O.; MacKerell Jr., A. D., FFParam: Standalone package for CHARMM additive and Drude polarizable force field parametrization of small molecules. J. Comput. Chem. 2019, $n / a(n / a)$.

32. Humphrey, W.; Dalke, A.; Schulten, K., VMD: Visual molecular dynamics. J. Mol. Graphics 1996, 14 (1), 33-38.

33. Lee, J.; Cheng, X.; Swails, J. M.; Yeom, M. S.; Eastman, P. K.; Lemkul, J. A.; Wei, S.; Buckner, J.; Jeong, J. C.; Qi, Y.; Jo, S.; Pande, V. S.; Case, D. A.; Brooks, C. L.; MacKerell, A. D.; Klauda, J. B.; Im, W., CHARMM-GUI Input Generator for NAMD, GROMACS, AMBER, OpenMM, and CHARMM/OpenMM Simulations Using the CHARMM36 Additive Force Field. J. Chem. Theory Comput. 2016, 12 (1), 405-413.

34. Tran, D. N. H.; Prime, E. L.; Plazzer, M.; Leung, A. H. M.; Yiapanis, G.; Christofferson, A. J.; Yarovsky, I.; Qiao, G. G.; Solomon, D. H., Molecular interactions behind the synergistic effect in mixed monolayers of 1-octadecanol and ethylene glycol monooctadecyl ether. Journal of Physical Chemistry B 2013, 117 (13), 3603-3612.

35. Yiapanis, G.; Christofferson, A. J.; Plazzer, M.; Weir, M.; Prime, E. L.; Qiao, G. G.; Solomon, D. H.; Yarovsky, l., Molecular mechanism of stabilization of thin films for improved water evaporation protection. Langmuir 2013, 29 (47), 14451-14459.

36. Tsimpanogiannis, I. N.; Moultos, O. A.; Franco, L. F. M.; Spera, M. B. d. M.; Erdős, M.; Economou, I. G., Self-diffusion coefficient of bulk and confined water: a critical review of classical molecular simulation studies. Mol. Simulat. 2019, 45 (4-5), 425-453.

37. Jorgensen, W. L.; Tirado-Rives, J., Potential energy functions for atomic-level simulations of water and organic and biomolecular systems. Proc. Natl. Acad. Sci. U.S.A. 2005, 102 (19), 6665.

38. Ouyang, J. F.; Bettens, R. P. A., Modelling Water: A Lifetime Enigma. CHIMIA International Journal for Chemistry 2015, 69 (3), 104-111.

39. Berendsen, H. J. C.; Postma, J. P. M.; Gunsteren, W. F. v.; Hermans, J., Interaction models for water in relation to protein hydration. In Intermolecular forces, Pullman, B., Ed. Reidel, Dordrecht, Holland: Springer, 1981; pp 331-342.

40. Schmid, N.; Eichenberger, A.; Choutko, A.; Riniker, S.; Winger, M.; Mark, A.; van Gunsteren, W., Definition and testing of the GROMOS force-field versions 54A7 and 54B7. Eur Biophys J 2011, 40 (7), 843-856.

41. Jorgensen, W. L.; Chandrasekhar, J.; Madura, J. D.; Impey, R. W.; Klein, M. L., Comparison of simple potential functions for simulating liquid water. J. Chem. Phys. 1983, 79 (2), 926-935.

42. Cornell, W. D.; Cieplak, P.; Bayly, C. I.; Gould, I. R.; Merz, K. M.; Ferguson, D. M.; Spellmeyer, D. C.; Fox, T.; Caldwell, J. W.; Kollman, P. A., A Second Generation Force Field for the Simulation of Proteins, Nucleic Acids, and Organic Molecules. J. Am. Chem. Soc. 1995, 117 (19), 5179-5197.

43. Jorgensen, W. L.; Maxwell, D. S.; Tirado-Rives, J., Development and Testing of the OPLS AllAtom Force Field on Conformational Energetics and Properties of Organic Liquids. J. Am. Chem. Soc. 1996, 118 (45), 11225-11236.

44. Sorenson, J. M.; Hura, G.; Glaeser, R. M.; Head-Gordon, T., What can x-ray scattering tell us about the radial distribution functions of water? J. Chem. Phys. 2000, 113 (20), 9149-9161.

45. III, W. E. R. Theoretical studies of hydrogen bonding. Harvard University, Cambridge, MA, USA, 1985.

46. MacKerell, A. D.; Bashford, D.; Bellott, M.; Dunbrack, R. L.; Evanseck, J. D.; Field, M. J.; Fischer, S.; Gao, J.; Guo, H.; Ha, S.; Joseph-McCarthy, D.; Kuchnir, L.; Kuczera, K.; Lau, F. T. K.; Mattos, C.; Michnick, S.; Ngo, T.; Nguyen, D. T.; Prodhom, B.; Reiher, W. E.; Roux, B.; Schlenkrich, 
M.; Smith, J. C.; Stote, R.; Straub, J.; Watanabe, M.; Wiórkiewicz-Kuczera, J.; Yin, D.; Karplus, M., All-Atom Empirical Potential for Molecular Modeling and Dynamics Studies of Proteins. J. Phys.

Chem. B 1998, 102 (18), 3586-3616.

47. Dauber-Osguthorpe, P.; Roberts, V. A.; Osguthorpe, D. J.; Wolff, J.; Genest, M.; Hagler, A. T., Structure and energetics of ligand binding to proteins: Escherichia coli dihydrofolate reductasetrimethoprim, a drug-receptor system. Proteins 1988, 4 (1), 31-47.

48. Whitelam, S.; Geissler, P. L., Avoiding unphysical kinetic traps in Monte Carlo simulations of strongly attractive particles. J. Chem. Phys. 2007, 127 (15), 154101.

49. Maple, J. R.; Hwang, M. J.; Stockfisch, T. P.; Dinur, U.; Waldman, M.; Ewig, C. S.; Hagler, A. T., Derivation Of Class II Force Fields .1. Methodology and Quantum Force Field for the Alkyl Functional Group and Alkane Molecules. J. Comput. Chem. 1994, 15, 162-182.

50. Sun, H., COMPASS: An ab Initio Force-Field Optimized for Condensed-Phase Applications_Overview with Details on Alkane and Benzene Compounds. Journal of Physical Chemistry B 1998, 102, 7338-7364.

51. Sun, H.; Mumby, S. J.; Maple, J. R.; Hagler, A. T., An ab Initio CFF93 All-Atom Force Field for Polycarbonates. J. Am. Chem. Soc. 1994, 116 (7), 2978-2987.

52. Chen, X. P.; Yuan, C. A.; Wong, C. K. Y.; Koh, S. W.; Zhang, G. Q., Validation of forcefields in predicting the physical and thermophysical properties of emeraldine base polyaniline. Mol. Simulat. 2011, 37 (12), 990-996.

53. Christofferson, A. J.; Yiapanis, G.; Ren, J. M.; Qiao, G. G.; Satoh, K.; Kamigaito, M.; Yarovsky, l., Molecular mapping of poly(methyl methacrylate) super-helix stereocomplexes. Chem. Sci. 2015, 6 (2), 1370-1378.

54. De Leener, C.; Hennebicq, E.; Sancho-Garcia, J.-C.; Beljonne, D., Modeling the Dynamics of Chromophores in Conjugated Polymers: The Case of Poly(2-methoxy-5-(2'-ethylhexyl)oxy 1,4phenylene vinylene) (MEH-PPV). J. Phys. Chem. B 2009, 113 (5), 1311-1322.

55. Díaz, I.; Díez, E.; Camacho, J.; León, S.; Ovejero, G., Comparison between three predictive methods for the calculation of polymer solubility parameters. Fluid Phase Equilib. 2013, 337 (0), 610.

56. Sun, H.; Jin, Z.; Yang, C.; Akkermans, R. L. C.; Robertson, S. H.; Spenley, N. A.; Miller, S.; Todd, S. M., COMPASS II: extended coverage for polymer and drug-like molecule databases. J. Mol. Model. 2016, 22 (2), 1-10.

57. Vega, C.; Abascal, J. L. F., Relation between the melting temperature and the temperature of maximum density for the most common models of water. J. Chem. Phys. 2005, 123 (14), 144504.

58. Mahoney, M. W.; Jorgensen, W. L., A five-site model for liquid water and the reproduction of the density anomaly by rigid, nonpolarizable potential functions. J. Chem. Phys. 2000, 112 (20), 89108922.

59. Berendsen, H. J. C.; Grigera, J. R.; Straatsma, T. P., The missing term in effective pair potentials. J. Phys. Chem. 1987, 91 (24), 6269-6271.

60. Wells, B. A.; Chaffee, A. L., Ewald Summation for Molecular Simulations. J. Chem. Theory Comput. 2015, 11 (8), 3684-3695.

61. Price, D. J.; Brooks, C. L., A modified TIP3P water potential for simulation with Ewald summation. J. Chem. Phys. 2004, 121 (20), 10096-10103.

62. Horn, H. W.; Swope, W. C.; Pitera, J. W.; Madura, J. D.; Dick, T. J.; Hura, G. L.; HeadGordon, T., Development of an improved four-site water model for biomolecular simulations: TIP4PEw. J. Chem. Phys. 2004, 120 (20), 9665-9678.

63. Rick, S. W., A reoptimization of the five-site water potential (TIP5P) for use with Ewald sums. J. Chem. Phys. 2004, 120 (13), 6085-6093.

64. Abascal, J. L. F.; Vega, C., A general purpose model for the condensed phases of water: TIP4P/2005. J. Chem. Phys. 2005, 123 (23), 234505.

65. Wu, Y.; Tepper, H. L.; Voth, G. A., Flexible simple point-charge water model with improved liquid-state properties. J. Chem. Phys. 2006, 124 (2), -. 
66. Schmitt, U. W.; Voth, G. A., The computer simulation of proton transport in water. J. Chem. Phys. 1999, 111 (20), 9361-9381.

67. González, M. A.; Abascal, J. L. F., A flexible model for water based on TIP4P/2005. J. Chem. Phys. 2011, 135 (22), 224516.

68. Lamoureux, G.; Harder, E.; Vorobyov, I. V.; Roux, B.; MacKerell Jr, A. D., A polarizable model of water for molecular dynamics simulations of biomolecules. Chem. Phys. Lett. 2006, 418 (13), 245-249.

69. Abascal, J. L. F.; Sanz, E.; García Fernández, R.; Vega, C., A potential model for the study of ices and amorphous water: TIP4P/Ice. J. Chem. Phys. 2005, 122 (23), 234511.

70. Fuentes-Azcatl, R.; Alejandre, J., Non-Polarizable Force Field of Water Based on the Dielectric Constant: TIP4P/ع. J. Phys. Chem. B 2014, 118 (5), 1263-1272.

71. Fuentes-Azcatl, R.; Mendoza, N.; Alejandre, J., Improved SPC force field of water based on the dielectric constant: SPC/E. Physica A: Statistical Mechanics and its Applications 2015, 420, 116123.

72. Fennell, C. J.; Li, L.; Dill, K. A., Simple Liquid Models with Corrected Dielectric Constants. J. Phys. Chem. B 2012, 116 (23), 6936-6944.

73. Fuentes-Azcatl, R.; Barbosa, M. C., Flexible bond and angle, FBA/ $\epsilon$ model of water. Journal of Molecular Liquids 2020, 303, 112598.

74. Takemura, K.; Kitao, A., Water Model Tuning for Improved Reproduction of Rotational Diffusion and NMR Spectral Density. J. Phys. Chem. B 2012, 116 (22), 6279-6287.

75. Piana, S.; Donchev, A. G.; Robustelli, P.; Shaw, D. E., Water Dispersion Interactions Strongly Influence Simulated Structural Properties of Disordered Protein States. J. Phys. Chem. B 2015, 119 (16), 5113-5123.

76. Wang, L.-P.; Martinez, T. J.; Pande, V. S., Building Force Fields: An Automatic, Systematic, and Reproducible Approach. J. Phys. Chem. Lett. 2014, 5 (11), 1885-1891.

77. Qiu, Y.; Nerenberg, P. S.; Head-Gordon, T.; Wang, L.-P., Systematic Optimization of Water Models Using Liquid/Vapor Surface Tension Data. J. Phys. Chem. B 2019, 123 (32), 7061-7073.

78. Khalak, Y.; Baumeier, B.; Karttunen, M., Improved general-purpose five-point model for water: TIP5P/2018. J. Chem. Phys. 2018, 149 (22), 224507.

79. Izadi, S.; Anandakrishnan, R.; Onufriev, A. V., Building Water Models: A Different Approach. J. Phys. Chem. Lett. 2014, 5 (21), 3863-3871.

80. Izadi, S.; Onufriev, A. V., Accuracy limit of rigid 3-point water models. J. Chem. Phys. 2016, 145 (7), 074501.

81. Dequidt, A.; Devémy, J.; Pádua, A. A. H., Thermalized Drude Oscillators with the LAMMPS Molecular Dynamics Simulator. J. Chem. Inf. Model 2016, 56 (1), 260-268.

82. Guillot, B., A reappraisal of what we have learnt during three decades of computer simulations on water. Journal of Molecular Liquids 2002, 101 (1), 219-260.

83. Onufriev, A., Chapter 7 - Implicit Solvent Models in Molecular Dynamics Simulations: A Brief Overview. In Annual Reports in Computational Chemistry, Wheeler, R. A.; Spellmeyer, D. C., Eds. Elsevier: 2008; Vol. 4, pp 125-137.

84. Anandakrishnan, R.; Izadi, S.; Onufriev, A. V., Why Computed Protein Folding Landscapes Are Sensitive to the Water Model. J. Chem. Theory Comput. 2019, 15 (1), 625-636.

85. Onufriev, A. V.; Izadi, S., Water models for biomolecular simulations. WIREs Comput. Mol. Sci. 2018, 8 (2), e1347.

86. Huan, Y. Q.; Liu, Y.; Goh, K. E. J.; Wong, S. L.; Lau, C. S., Deep learning-enabled prediction of 2D material breakdown. Nanotechnology 2021, 32 (26), 265203.

87. Meftahi, N.; Klymenko, M.; Christofferson, A. J.; Bach, U.; Winkler, D. A.; Russo, S. P., Machine learning property prediction for organic photovoltaic devices. npj Comput. Mater. 2020, 6 (1), 166.

88. Le, T. C.; Winkler, D. A., Applications in Materials Science. In Applied Chemoinformatics, 2018; pp 547-569. 
89. Tawfik, S. A.; Isayev, O.; Stampfl, C.; Shapter, J.; Winkler, D. A.; Ford, M. J., Efficient Prediction of Structural and Electronic Properties of Hybrid 2D Materials Using Complementary DFT and Machine Learning Approaches. Adv. Theory Simul. 2019, 2 (1), 1800128.

90. Koutsoukos, S.; Philippi, F.; Malaret, F.; Welton, T., A review on machine learning algorithms for the ionic liquid chemical space. Chem. Sci. 2021.

91. Meftahi, N.; Walker, M. L.; Enciso, M.; Smith, B. J., Predicting the Enthalpy and Gibbs Energy of Sublimation by QSPR Modeling. Sci. Rep. 2018, 8 (1), 9779.

92. Meftahi, N.; Walker, M. L.; Smith, B. J., Predicting aqueous solubility by QSPR modeling. J. Mol. Graph. Model. 2021, 106, 107901.

93. Sankar, K.; Hoi, K. H.; Yin, Y.; Ramachandran, P.; Andersen, N.; Hilderbrand, A.; McDonald, P.; Spiess, C.; Zhang, Q., Prediction of methionine oxidation risk in monoclonal antibodies using a machine learning method. mAbs 2018, 10 (8), 1281-1290.

94. Chen, X.; Xie, W.; Yang, Y.; Hua, Y.; Xing, G.; Liang, L.; Deng, C.; Wang, Y.; Fan, Y.; Liu, H.; Lu, T.; Chen, Y.; Zhang, Y., Discovery of Dual FGFR4 and EGFR Inhibitors by Machine Learning and Biological Evaluation. J. Chem. Inf. Model 2020, 60 (10), 4640-4652.

95. Ghasemi, J. B.; Meftahi, N., Docking, CoMFA and CoMSIA studies of a series of sulfonamides derivatives as carbonic anhydrase I inhibitors. J. Enzyme Inhib. Med. Chem. 2013, 28 (2), 320-327.

96. Ghasemi, J. B.; Meftahi, N.; Pirhadi, S.; Tavakoli, H., Docking and pharmacophore-based alignment comparative molecular field analysis three-dimensional quantitative structure-activity relationship analysis of dihydrofolate reductase inhibitors by linear and nonlinear calibration methods. J. Chemometrics 2013, 27 (10), 287-296.

97. Kasabe, A. J.; Kulkarni, A. S.; Gaikwad, V. L., QSPR Modeling of Biopharmaceutical Properties of Hydroxypropyl Methylcellulose (Cellulose Ethers) Tablets Based on Its Degree of Polymerization. AAPS PharmSciTech 2019, 20 (8), 308.

98. Phase, Schrödinger, LLC, New York, NY, 2021.

99. Tripos Inc., St. Louis, USA.

100. Abadi, M.; Barham, P.; Chen, J.; Chen, Z.; Davis, A.; Dean, J.; Devin, M.; Ghemawat, S.; Irving, G.; Isard, M. In Tensorflow: A system for large-scale machine learning, 12th \{USENIX\} symposium on operating systems design and implementation (\{OSDI\} 16), 2016; pp 265-283.

101. Enciso, M.; Meftahi, N.; Walker, M. L.; Smith, B. J., BioPPSy: An Open-Source Platform for QSAR/QSPR Analysis. PLOS ONE 2016, 11 (11), e0166298.

102. Plimpton, S., Fast Parallel Algorithms for Short-Range Molecular Dynamics. J. Comput. Phys. 1995, $117(1), 1-19$.

103. Padua, A. A Tool to Build Force Field Input Files for Molecular Dynamics, https://github.com/paduagroup/fftool, 2015.

104. Humbert, M. T.; Zhang, Y.; Maginn, E. J., PyLAT: Python LAMMPS Analysis Tools. J. Chem. Inf. Model 2019, 59 (4), 1301-1305.

105. Martínez, L.; Andrade, R.; Birgin, E. G.; Martínez, J. M., PACKMOL: A package for building initial configurations for molecular dynamics simulations. J. Comput. Chem. 2009, 30 (13), 21572164.

106. Kohlmeyer, A. TopoTools: Release 1.7, 2016.

107. Allen, M. P.; Tildesley, D. J., Computer Simulation of Liquids. Oxford University Press: Oxford, U.K., 2017.

108. Vega, C.; Miguel, E. d., Surface tension of the most popular models of water by using the test-area simulation method. J. Chem. Phys. 2007, 126 (15), 154707.

109. Widom, B., Some Topics in the Theory of Fluids. J. Chem. Phys. 1963, 39 (11), 2808-2812.

110. Paschek, D., Temperature dependence of the hydrophobic hydration and interaction of simple solutes: An examination of five popular water models. J. Chem. Phys. 2004, 120 (14), 66746690.

111. Liu, M.; Besford, Q. A.; Mulvaney, T.; Gray-Weale, A., Order and correlation contributions to the entropy of hydrophobic solvation. J. Chem. Phys. 2015, 142 (11), 114117. 
112. Hansch, C.; Maloney, P. P.; Fujita, T.; Muir, R. M., Correlation of biological activity of phenoxyacetic acids with Hammett substituent constants and partition coefficients. Nature 1962, 194 (4824), 178-180.

113. Figueiredo, M. A. T., Adaptive sparseness for supervised learning. IEEE Transactions on Pattern Analysis and Machine Intelligence 2003, 25 (9), 1150-1159.

114. Burden, F. R.; Winkler, D. A., New QSAR methods applied to structure- activity mapping and combinatorial chemistry. J. Chem. Inf. Comput. Sci. 1999, 39 (2), 236-242.

115. Burden, F. R.; Winkler, D. A., Robust QSAR models using Bayesian regularized neural networks. J. Med. Chem. 1999, 42 (16), 3183-3187.

116. Winkler, D. A.; Burden, F. R., Robust QSAR models from novel descriptors and Bayesian regularised neural networks. Mol. Simulat. 2000, 24 (4-6), 243-258.

117. Burden, F.; Winkler, D., Optimal sparse descriptor selection for QSAR using Bayesian methods. QSAR Comb. Sci. 2009, 28 (6-7), 645-653.

118. Burden, F. R.; Winkler, D. A., An Optimal Self-Pruning Neural Network and Nonlinear Descriptor Selection in QSAR. QSAR Comb. Sci. 2009, 28 (10), 1092-1097.

119. Alexander, D. L.; Tropsha, A.; Winkler, D. A., Beware of R 2: simple, unambiguous assessment of the prediction accuracy of QSAR and QSPR models. J. Chem. Inf. Model 2015, 55 (7), 1316-1322.

120. CRC Handbook of Chemistry and Physics. 96th ed.; CRC Press: 2015.

121. Head-Gordon, T.; Johnson, M. E., Tetrahedral structure or chains for liquid water.

Proceedings of the National Academy of Sciences 2006, 103 (21), 7973.

122. Pallas, N. R.; Harrison, Y., An automated drop shape apparatus and the surface tension of pure water. Colloids and Surfaces 1990, 43 (2), 169-194.

123. Malmberg, C. G.; Maryott, A. A., Dielectric Constant of Water from 0 to 100 C. Journal of Research of the National Bureau of Standards 1956, 56 (1), 1.

124. Pruppacher, H. R., Self-Diffusion Coefficient of Supercooled Water. J. Chem. Phys. 1972, 56 (1), 101-107.

125. Ben-Naim, A.; Marcus, Y., Solvation thermodynamics of nonionic solutes. J. Chem. Phys. 1984, 81 (4), 2016-2027.

126. Hockney, R. W.; Eastwood, J. W., Computer Simulation Using Particles. Adam Hilger: New York, 1989.

127. Darden, T.; York, D.; Pedersen, L., Particle mesh Ewald: An N· $\log (\mathrm{N})$ method for Ewald sums in large systems. J. Chem. Phys. 1993, 98 (12), 10089-10092.

128. Rigby, D., Fluid density predictions using the COMPASS force field. Fluid Phase Equilib. 2004, $217(1), 77-87$.

129. Maple, J. R.; Dinur, U.; Hagler, A. T., Derivation of force fields for molecular mechanics and dynamics from ab initio energy surfaces. Proceedings of the National Academy of Sciences 1988, 85 (15), 5350-5354.

130. Salas, F. J.; Méndez-Maldonado, G. A.; Núñez-Rojas, E.; Aguilar-Pineda, G. E.; Domínguez, H.; Alejandre, J., Systematic Procedure To Parametrize Force Fields for Molecular Fluids. J. Chem. Theory Comput. 2015, 11 (2), 683-693.

131. Sega, M.; Dellago, C., Long-Range Dispersion Effects on the Water/Vapor Interface Simulated Using the Most Common Models. J. Phys. Chem. B 2017, 121 (15), 3798-3803.

132. Besford, Q. A.; Liu, M.; Christofferson, A. J., Stabilizing Dipolar Interactions Drive Specific Molecular Structure at the Water Liquid-Vapor Interface. Journal of Physical Chemistry B 2018, 122 (34), 8309-8314.

133. Besford, Q. A.; Christofferson, A. J.; Liu, M.; Yarovsky, I., Long-range dipolar order and dispersion forces in polar liquids. J. Chem. Phys. 2017, 147 (19), 194503.

134. Neumann, M., The dielectric constant of water. Computer simulations with the MCY potential. J. Chem. Phys. 1985, 82 (12), 5663-5672. 
135. Kusalik, P. G.; Svishchev, I. M., The Spatial Structure in Liquid Water. Science 1994, 265 (5176), 1219.

136. Dang, L. X.; Pettitt, B. M., A theoretical study of like ion pairs in solution. J. Phys. Chem. 1990, 94 (10), 4303-4308.

137. Simonson, T., Accurate calculation of the dielectric constant of water from simulations of a microscopic droplet in vacuum. Chem. Phys. Lett. 1996, 250 (5), 450-454.

138. Discover molecular mechanics and dynmics simulation package, Biosym Technologies, Inc.: 10065 Barnes Canyon Road, Suite A, San Diego, CA 92121.

139. Lau, K. F.; Alper, H. E.; Thacher, T. S.; Stouch, T. R., Effects of Switching Functions on the Behavior of Liquid Water in Molecular Dynamics Simulations. J. Phys. Chem. 1994, 98 (35), 87858792.

140. Guo, J.; Tardy, B. L.; Christofferson, A. J.; Dai, Y.; Richardson, J. J.; Zhu, W.; Hu, M.; Ju, Y.; Cui, J.; Dagastine, R. R.; Yarovsky, I.; Caruso, F., Modular assembly of superstructures from polyphenol-functionalized building blocks. Nat. Nanotechnol. 2016, 11 (12), 1105-1111.

141. Guo, J.; Richardson, J. J.; Besford, Q. A.; Christofferson, A. J.; Dai, Y.; Ong, C. W.; Tardy, B. L.; Liang, K.; Choi, G. H.; Cui, J.; Yoo, P. J.; Yarovsky, I.; Caruso, F., Influence of lonic Strength on the Deposition of Metal-Phenolic Networks. Langmuir 2017, 33 (40), 10616-10622.

142. Herpoldt, K.-L.; Artzy-Schnirman, A.; Christofferson, A. J.; Makarucha, A. J.; de la Rica, R.; Yarovsky, I.; Stevens, M. M., Designing Fluorescent Peptide Sensors with Dual Specificity for the Detection of HIV-1 Protease. Chem. Mater. 2015, 27 (20), 7187-7195.

143. Wright, L. B.; Walsh, T. R., Efficient conformational sampling of peptides adsorbed onto inorganic surfaces: insights from a quartz binding peptide. Phys. Chem. Chem. Phys. 2013, 15 (13), 4715-4726.

144. Bernardi, R. C.; Melo, M. C. R.; Schulten, K., Enhanced sampling techniques in molecular dynamics simulations of biological systems. Biochimica et Biophysica Acta (BBA) - General Subjects 2014, 1850 (5), 872-877.

145. Yeh, I.-C.; Hummer, G., System-Size Dependence of Diffusion Coefficients and Viscosities from Molecular Dynamics Simulations with Periodic Boundary Conditions. J. Phys. Chem. B 2004, 108 (40), 15873-15879.

146. Mobley, D. L.; Bayly, C. I.; Cooper, M. D.; Shirts, M. R.; Dill, K. A., Small Molecule Hydration Free Energies in Explicit Solvent: An Extensive Test of Fixed-Charge Atomistic Simulations. J. Chem. Theory Comput. 2009, 5 (2), 350-358.

147. Raabe, G.; Sadus, R. J., Molecular dynamics simulation of the dielectric constant of water: The effect of bond flexibility. J. Chem. Phys. 2011, 134 (23), 234501.

148. Raabe, G.; Sadus, R. J., Molecular dynamics simulation of the effect of bond flexibility on the transport properties of water. J. Chem. Phys. 2012, 137 (10), 104512.

149. Vega, C.; Abascal, J. L. F., Simulating water with rigid non-polarizable models: a general perspective. Phys. Chem. Chem. Phys. 2011, 13 (44), 19663-19688.

150. Huang, J.; Rauscher, S.; Nawrocki, G.; Ran, T.; Feig, M.; de Groot, B. L.; Grubmüller, H.; MacKerell, A. D., CHARMM36m: an improved force field for folded and intrinsically disordered proteins. Nat. Methods 2017, 14 (1), 71-73.

151. Ren, J. M.; Satoh, K.; Goh, T. K.; Blencowe, A.; Nagai, K.; Ishitake, K.; Christofferson, A. J.; Yiapanis, G.; Yarovsky, I.; Kamigaito, M.; Qiao, G. G., Stereospecific Cyclic Poly(methyl methacrylate) and Its Topology-Guided Hierarchically Controlled Supramolecular Assemblies. Angew. Chem. Int. Ed. 2014, 53 (2), 459-464.

152. Christofferson, A. J.; Yiapanis, G.; Leung, A. H. M.; Prime, E. L.; Tran, D. N. H.; Qiao, G. G.; Solomon, D. H.; Yarovsky, I., Dynamic Performance of Duolayers at the Air/Water Interface. 2. Mechanistic Insights from All-Atom Simulations. J. Phys. Chem. B 2014, 118 (37), 10927-10933. 153. Shabane, P. S.; Izadi, S.; Onufriev, A. V., General Purpose Water Model Can Improve Atomistic Simulations of Intrinsically Disordered Proteins. J. Chem. Theory Comput. 2019, 15 (4), 2620-2634. 
154. Zhang, H.; Yin, C.; Jiang, Y.; van der Spoel, D., Force Field Benchmark of Amino Acids: I. Hydration and Diffusion in Different Water Models. J. Chem. Inf. Model 2018, 58 (5), 1037-1052. 155. Giri, A. K.; Spohr, E., Cluster formation of $\mathrm{NaCl}$ in bulk solutions: Arithmetic vs. geometric combination rules. Journal of Molecular Liquids 2017, 228, 63-70.

156. Sengupta, A.; Li, Z.; Song, L. F.; Li, P.; Merz, K. M., Parameterization of Monovalent lons for the OPC3, OPC, TIP3P-FB, and TIP4P-FB Water Models. J. Chem. Inf. Model 2021, 61 (2), 869-880.

157. Li, Z.; Song, L. F.; Li, P.; Merz, K. M., Parametrization of Trivalent and Tetravalent Metal lons for the OPC3, OPC, TIP3P-FB, and TIP4P-FB Water Models. J. Chem. Theory Comput. 2021, 17 (4), 2342-2354.

158. Li, Z.; Song, L. F.; Li, P.; Merz, K. M., Systematic Parametrization of Divalent Metal lons for the OPC3, OPC, TIP3P-FB, and TIP4P-FB Water Models. J. Chem. Theory Comput. 2020, 16 (7), 44294442.

159. Vanommeslaeghe, K.; MacKerell, A. D., CHARMM additive and polarizable force fields for biophysics and computer-aided drug design. Biochimica et Biophysica Acta (BBA) - General Subjects 2015, 1850 (5), 861-871.

160. Tian, C.; Kasavajhala, K.; Belfon, K. A. A.; Raguette, L.; Huang, H.; Migues, A. N.; Bickel, J.; Wang, Y.; Pincay, J.; Wu, Q.; Simmerling, C., ff19SB: Amino-Acid-Specific Protein Backbone Parameters Trained against Quantum Mechanics Energy Surfaces in Solution. J. Chem. Theory Comput. 2020, 16 (1), 528-552.

161. Zhao, C.-L.; Zhao, D.-X.; Bei, C.-C.; Meng, X.-N.; Li, S.; Yang, Z.-Z., Seven-Site Effective Pair Potential for Simulating Liquid Water. J. Phys. Chem. B 2019, 123 (21), 4594-4603.

162. Zhang, W.; van Duin, A. C. T., Second-Generation ReaxFF Water Force Field: Improvements in the Description of Water Density and OH-Anion Diffusion. J. Phys. Chem. B 2017, 121 (24), 60216032.

163. Hughes, Z. E.; Ren, E.; Thacker, J. C. R.; Symons, B. C. B.; Silva, A. F.; Popelier, P. L. A., A FFLUX Water Model: Flexible, Polarizable and with a Multipolar Description of Electrostatics. J. Comput. Chem. 2020, 41 (7), 619-628.

164. Calio, P. B.; Hocky, G. M.; Voth, G. A., Minimal Experimental Bias on the Hydrogen Bond Greatly Improves Ab Initio Molecular Dynamics Simulations of Water. J. Chem. Theory Comput. 2020, 16 (9), 5675-5684.

165. Zhang, L.; Wang, H.; Car, R.; E, W., The Phase Diagram of a Deep Potential Water Model. arXiv 2021, https://arxiv.org/abs/2102.04804v3.

166. Dawson, W.; Gygi, F., Equilibration and analysis of first-principles molecular dynamics simulations of water. J. Chem. Phys. 2018, 148 (12), 124501.

167. Jin, J.; Han, Y.; Pak, A. J.; Voth, G. A., A new one-site coarse-grained model for water: Bottom-up many-body projected water (BUMPer). I. General theory and model. J. Chem. Phys. 2021, 154 (4), 044104.

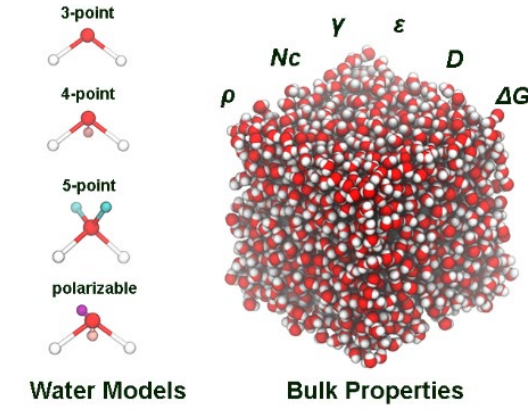

For Table of Contents Only

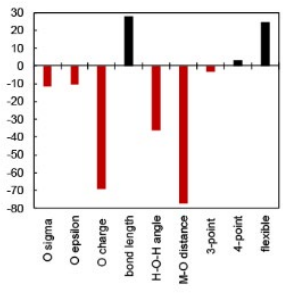

Machine Learning
Analysis 
Supporting Information for

\section{A systematic comparison of the structural and dynamic properties of commonly used water models for molecular dynamics simulations}

Sachini P. Kadaoluwa Pathirannahalage, ${ }^{\mathrm{a}, \mathrm{b}, \dagger}$ Nastaran Meftahi, ${ }^{\mathrm{c}, \dagger}$ Aaron Elbourne, ${ }^{\mathrm{a}}$ Alessia C. G.

Weiss, ${ }^{\mathrm{d}}$ Chris F. McConville, ${ }^{\mathrm{a} e \mathrm{e}}$ Agilio Padua, ${ }^{\mathrm{b}}$ David A. Winkler, ${ }^{\mathrm{f}}$ Margarida Costa Gomes, ${ }^{\mathrm{b}}$ Tamar L. Greaves, ${ }^{a}$ Tu C. Le, ${ }^{\mathrm{g}, *}$ Quinn A. Besford, ${ }^{\mathrm{d}, *}$ and Andrew J. Christofferson ${ }^{\mathrm{a}, *}$

${ }^{\text {a }}$ School of Science, RMIT University, Melbourne, Victoria 3000, Australia

${ }^{\mathrm{b}}$ Laboratoire de Chimie, Ecole Normale Supérieure de Lyon and CNRS, Lyon, France

c ARC Centre of Excellence in Exciton Science, School of Science, RMIT University, Melbourne, Victoria 3000, Australia

${ }^{\mathrm{d}}$ Leibniz Institute für Polymerforschung e.V., Hohe Straße 6, 01069 Dresden, Germany

${ }^{\mathrm{e}}$ Institute for Frontier Materials, Deakin University, Geelong, Victoria 3220, Australia

${ }^{\mathrm{f}}$ School of Biochemistry and Genetics, La Trobe Institute for Molecular Science, La Trobe University, Bundoora, VIC 3086, Australia

Monash Institute of Pharmaceutical Sciences, Monash University, Parkville, VIC 3052, Australia School of Pharmacy, University of Nottingham, Nottingham NG7 2QL, UK

CSIRO Data61, Pullenvale, QLD 4069, Australia

g School of Engineering, RMIT University, Melbourne, Victoria 3001, Australia

*Corresponding authors: tu.le@rmit.edu.au, besford@ipfdd.de, andrew.christofferson@rmit.edu.au

$\dagger$ These authors contributed equally 
The functional form of the Lennard-Jones 12-6 potential is

$$
U_{12-6}(r)=4 \varepsilon\left[\left(\frac{\sigma}{r}\right)^{12}-\left(\frac{\sigma}{r}\right)^{6}\right]
$$

whereas that for the Lennard-Jones 9-6 potential is

$$
U_{9-6}(r)=\varepsilon\left[2\left(\frac{\sigma}{r}\right)^{9}-3\left(\frac{\sigma}{r}\right)^{6}\right]
$$

where $r$ is the distance between interacting atoms, $\varepsilon$ (epsilon) describes the depth of the attractive well, and $\sigma$ (sigma) the inter-atom distance where the potential changes sign. Some papers report $r_{\min }$ rather than $\sigma$, where $r_{\min }=\sigma \times 2^{1 / 6}$.
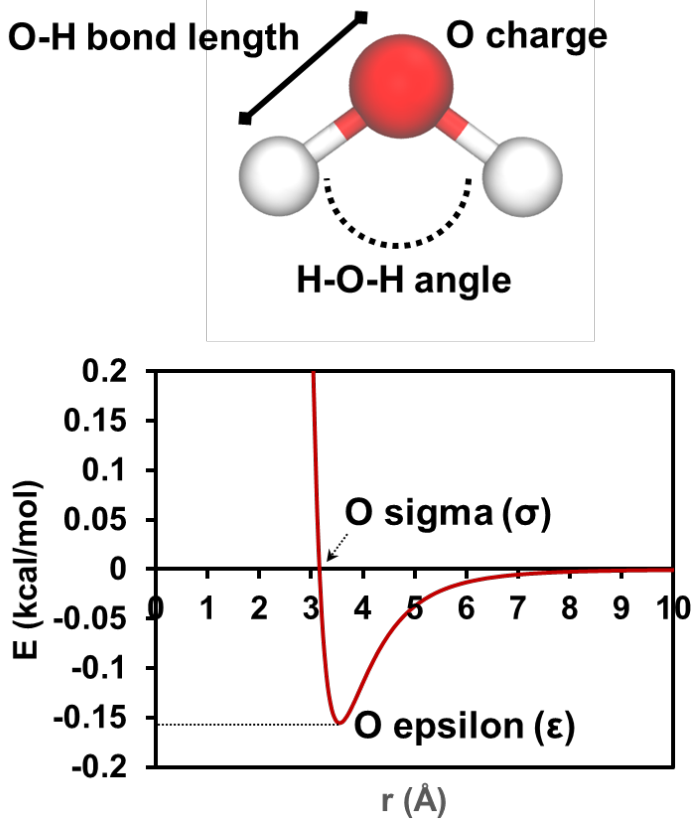

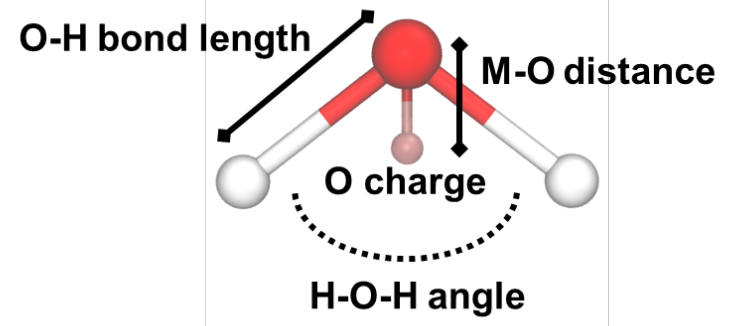

\begin{tabular}{|c|c|c|c|c|c|}
\hline \multicolumn{4}{|c|}{ 1-hot binary descriptors } \\
\hline 3-point? & \multicolumn{2}{|c|}{ 4-point? } & \multicolumn{2}{|c|}{ Flexible? } \\
\hline Yes & No & Yes & No & Yes & No \\
\hline 1 & 0 & 1 & 0 & 1 & 0 \\
\hline
\end{tabular}

Figure S1. A schematic of the descriptors used for machine learning. 


\begin{tabular}{|c|c|c|c|c|c|c|}
\hline & Density & $\frac{\text { Coordination }}{\text { number }}$ & $\frac{\text { Surface }}{\text { Tension }}$ & $\frac{\text { Dielectric }}{\text { constant }}$ & $\frac{\text { Self-diffusion }}{\text { coefficient }}$ & $\frac{\text { Average difference }}{\text { from experiment }}$ \\
\hline \multicolumn{7}{|l|}{ 1980s } \\
\hline SPC & $2.5 \%$ & $19 \%$ & $30 \%$ & $17 \%$ & $88 \%$ & $31 \%$ \\
\hline TIP3P & $1.7 \%$ & $33 \%$ & $35 \%$ & $22 \%$ & $149 \%$ & $48 \%$ \\
\hline TIP4P & $0.3 \%$ & $9.4 \%$ & $27 \%$ & $35 \%$ & $12 \%$ & $17 \%$ \\
\hline TIPS3P & $1.0 \%$ & $60 \%$ & $29 \%$ & $35 \%$ & $142 \%$ & $53 \%$ \\
\hline SPC/E & $0.4 \%$ & $4.3 \%$ & $20 \%$ & $6.5 \%$ & $13 \%$ & $8.9 \%$ \\
\hline CVFF & $1.9 \%$ & $36 \%$ & $34 \%$ & $72 \%$ & $159 \%$ & $61 \%$ \\
\hline $\operatorname{CVFF}(\mathrm{cm})$ & $0.8 \%$ & $23 \%$ & $31 \%$ & $93 \%$ & $126 \%$ & $55 \%$ \\
\hline \multicolumn{7}{|l|}{$1990 s$} \\
\hline PCFF & $1.1 \%$ & $187 \%$ & $3.5 \%$ & $103 \%$ & $236 \%$ & $106 \%$ \\
\hline COMPASS & $3.9 \%$ & $5.4 \%$ & $43 \%$ & $50 \%$ & $180 \%$ & $57 \%$ \\
\hline TIP3P/FW & $3.0 \%$ & $13 \%$ & $23 \%$ & $152 \%$ & $67 \%$ & $52 \%$ \\
\hline \multicolumn{7}{|l|}{$2000 s$} \\
\hline TIP5P & $1.2 \%$ & $6.2 \%$ & $32 \%$ & $20 \%$ & $22 \%$ & $16 \%$ \\
\hline TIP3P-Ew & $0.1 \%$ & $0.6 \%$ & $35 \%$ & $17 \%$ & $79 \%$ & $26 \%$ \\
\hline TIP4P-Ew & $0.1 \%$ & $0.2 \%$ & $18 \%$ & $17 \%$ & $10 \%$ & $9.0 \%$ \\
\hline TIP5P-Ew & $0.6 \%$ & $9.4 \%$ & $27 \%$ & $28 \%$ & $27 \%$ & $18 \%$ \\
\hline TIP4P/2005 & $0 \%$ & $0.1 \%$ & $12 \%$ & $26 \%$ & $5.3 \%$ & $8.6 \%$ \\
\hline TIP4P/Ice & $0.4 \%$ & $1.4 \%$ & $2.0 \%$ & $19 \%$ & $47 \%$ & $14 \%$ \\
\hline $\mathrm{SPC} / \mathrm{Fw}$ & $1.0 \%$ & $0.9 \%$ & $19 \%$ & $1.9 \%$ & $12 \%$ & $6.8 \%$ \\
\hline SWM4-NDP & $0.7 \%$ & $11 \%$ & $12 \%$ & $4.5 \%$ & $12 \%$ & $8.1 \%$ \\
\hline \multicolumn{7}{|l|}{ 2010s } \\
\hline TIP4P/2005f & $0.1 \%$ & $6.4 \%$ & $16 \%$ & $25 \%$ & $20 \%$ & $14 \%$ \\
\hline TIP4P/ع & $0.1 \%$ & $0.4 \%$ & $10 \%$ & $0.7 \%$ & $6.0 \%$ & $3.5 \%$ \\
\hline OPC & $0 \%$ & $11 \%$ & $2.6 \%$ & $0.1 \%$ & $1.1 \%$ & $2.9 \%$ \\
\hline TIP3P-FB & $0.7 \%$ & $0.5 \%$ & $16 \%$ & $1.1 \%$ & $7.1 \%$ & $5.1 \%$ \\
\hline TIP4P-FB & $0 \%$ & $0.04 \%$ & $10 \%$ & $2.1 \%$ & $8.7 \%$ & $4.2 \%$ \\
\hline TIP4P-D & $0.4 \%$ & $9.6 \%$ & $1.7 \%$ & $19 \%$ & $12 \%$ & $8.6 \%$ \\
\hline $\mathrm{SPC} / \varepsilon$ & $0.6 \%$ & $0.6 \%$ & $9.3 \%$ & $2.0 \%$ & $33 \%$ & $9.0 \%$ \\
\hline OPC3 & $0.6 \%$ & $4.3 \%$ & $15.3 \%$ & $0.8 \%$ & $0.7 \%$ & $4.3 \%$ \\
\hline a99SB-disp & $0.1 \%$ & $10 \%$ & $3.3 \%$ & $15 \%$ & $22 \%$ & $10 \%$ \\
\hline TIP5P-2018 & $0 \%$ & $10 \%$ & $14 \%$ & $65 \%$ & $0.6 \%$ & $18 \%$ \\
\hline TIP3P-ST & $0.4 \%$ & $2.1 \%$ & $11 \%$ & $3.3 \%$ & $46 \%$ & $13 \%$ \\
\hline TIP4P-ST & $0.2 \%$ & $0.1 \%$ & $10 \%$ & $4.3 \%$ & $12 \%$ & $5.4 \%$ \\
\hline \multicolumn{7}{|l|}{2020 s } \\
\hline $\mathrm{FBA} / \varepsilon$ & $0.6 \%$ & $1.3 \%$ & $5.5 \%$ & $4.5 \%$ & $32 \%$ & $8.8 \%$ \\
\hline
\end{tabular}

Figure S2. Color gradient depiction of the percent difference of the calculated value compared to the experimental value for the calculated structural and dynamic properties of each model. The minimum, maximum, and mean values are colored green, yellow, and red, respectively. 

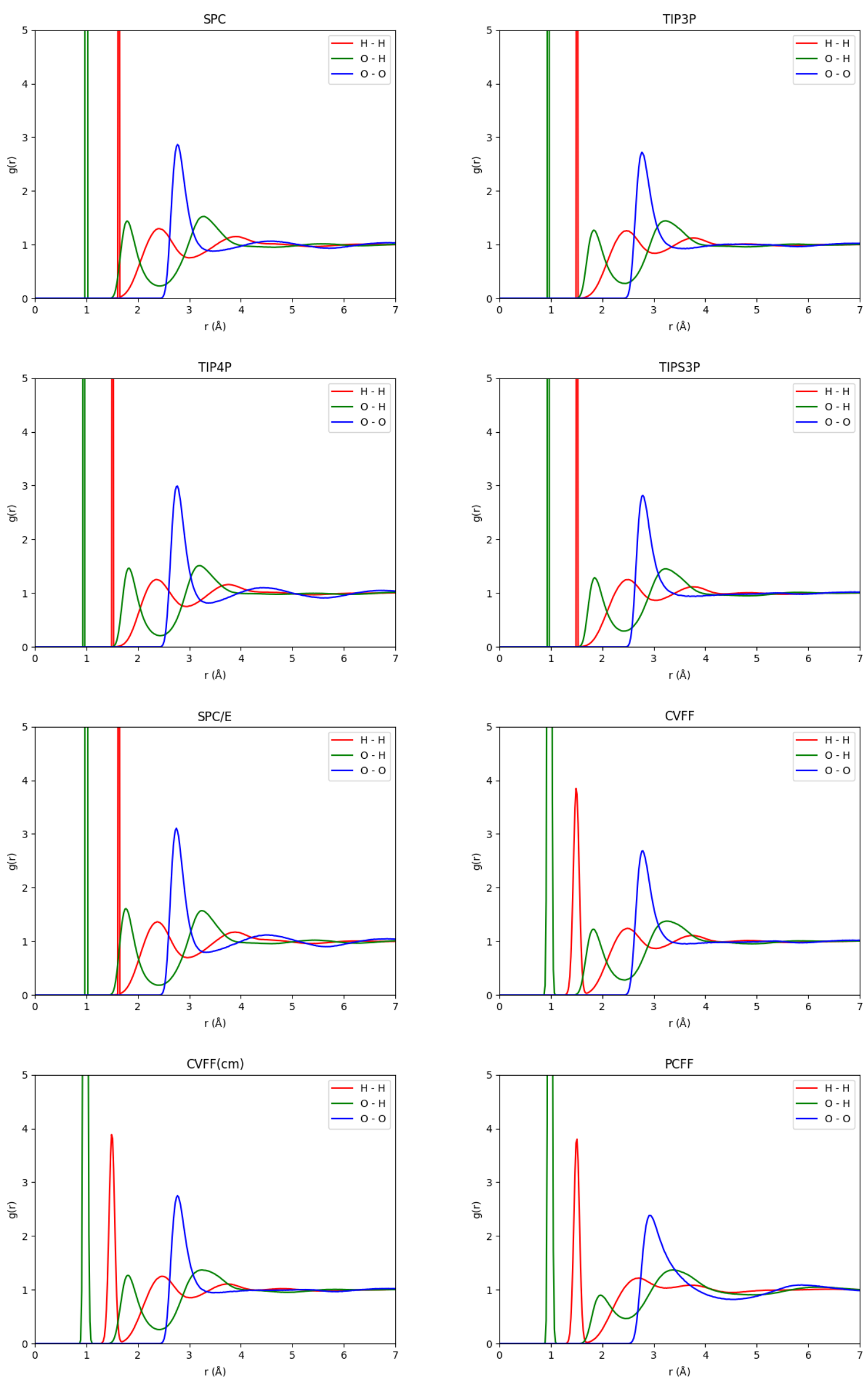

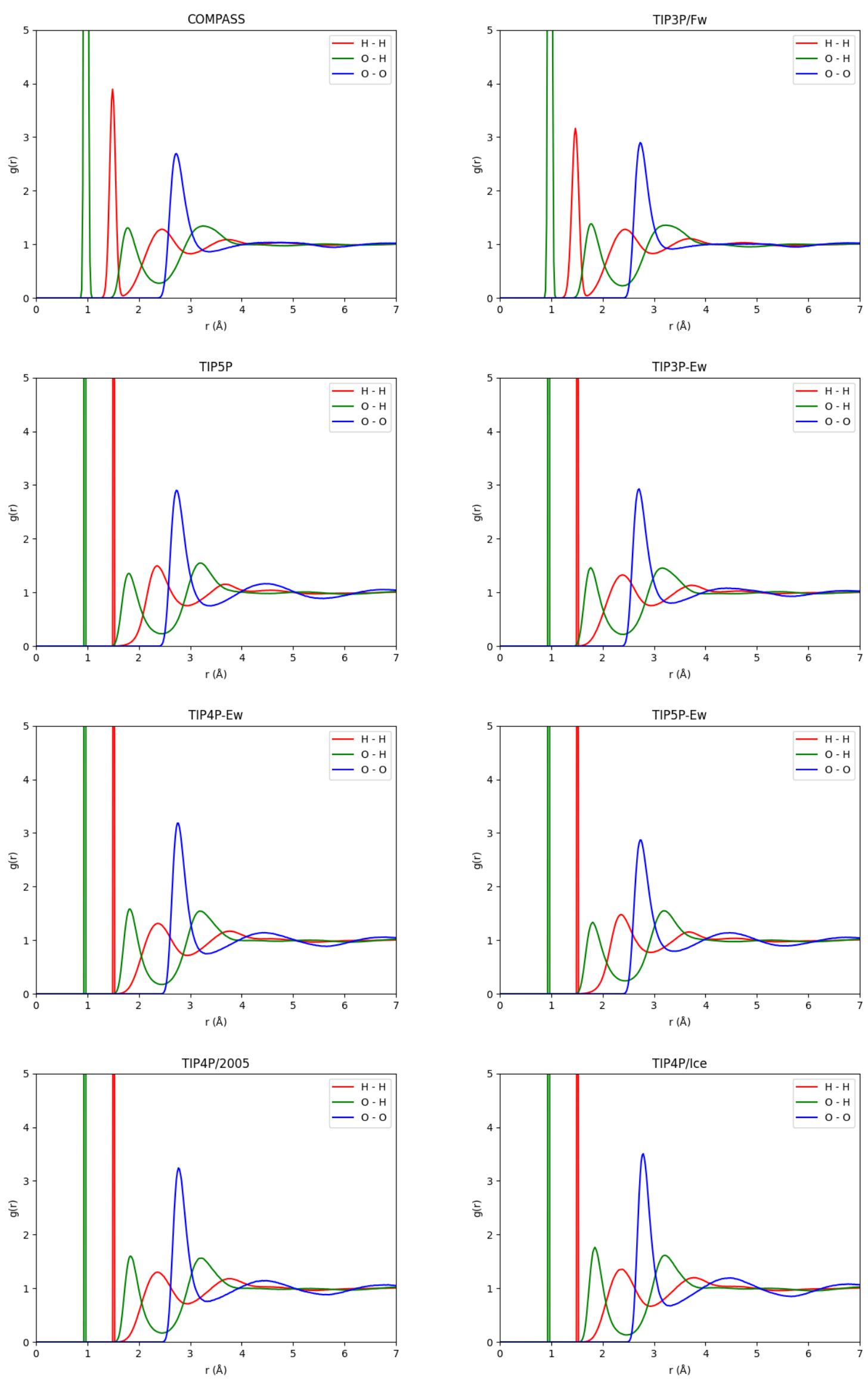

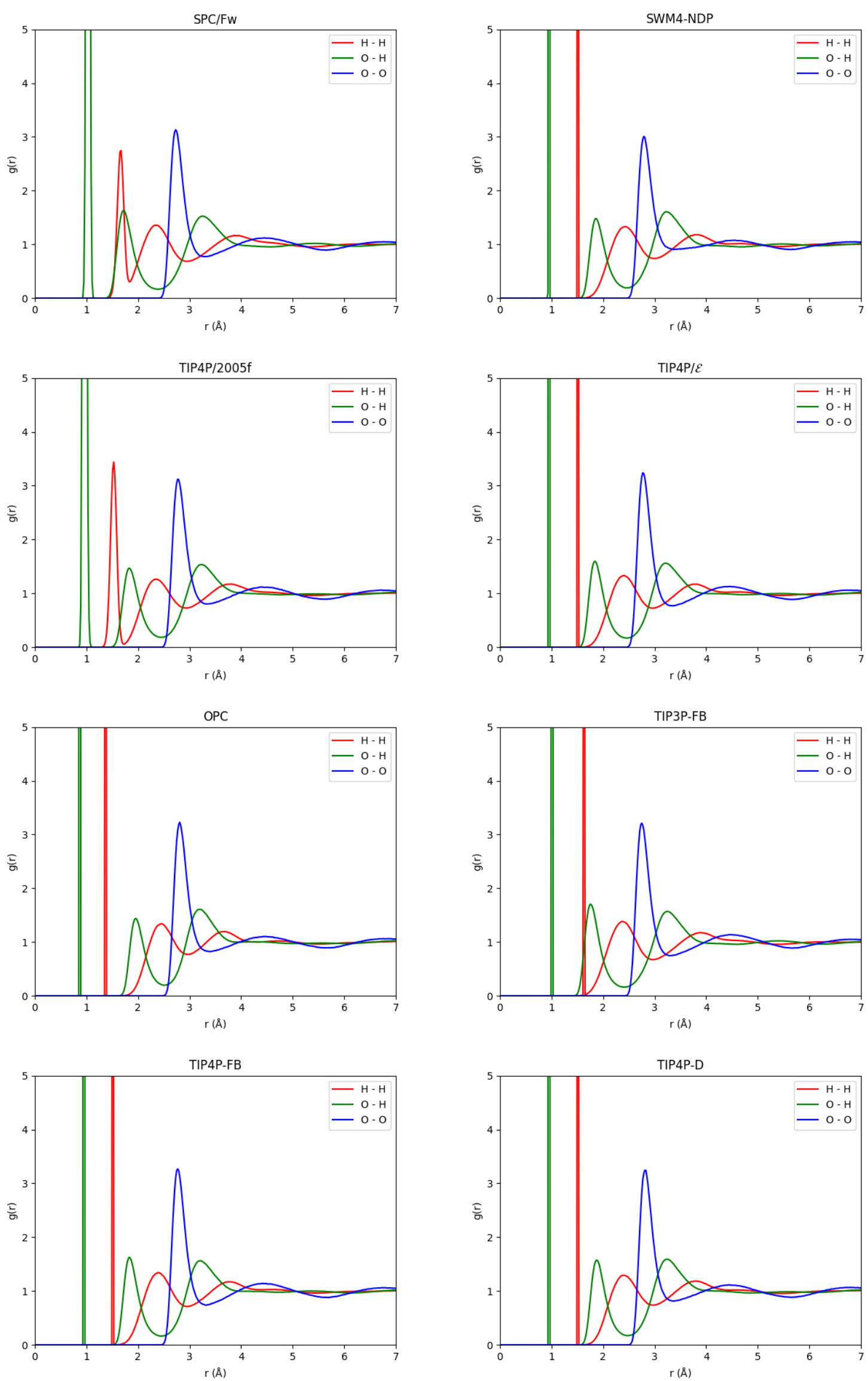

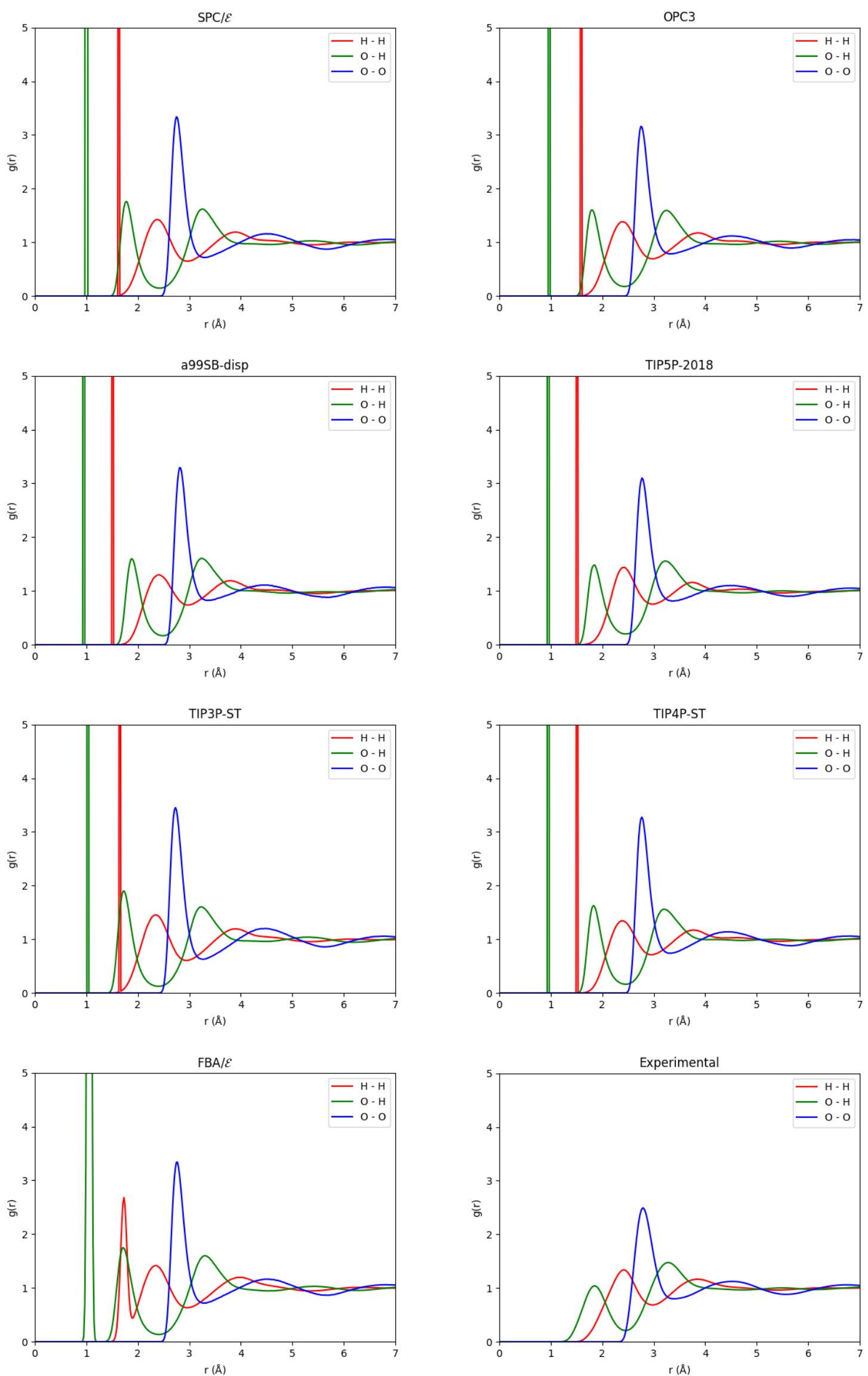

Figure S3. Radial distribution functions (RDFs) for the water models examined in this work. The experimental RDFs are taken from: https://doi.org/10.1155/2013/279463. 